\title{
CRITICAL LIL BEHAVIOR OF THE TRIGONOMETRIC SYSTEM
}

\author{
I. BERKES
}

\begin{abstract}
It is a classical fact that for rapidly increasing $\left(n_{k}\right)$ the sequence $\left(\cos n_{k} x\right)$ behaves like a sequence of i.i.d. random variables. Actually, this almost i.i.d. behavior holds if $\left(n_{k}\right)$ grows faster than $e^{c \sqrt{k}}$; below this speed we have strong dependence. While there is a large literature dealing with the almost i.i.d. case, practically nothing is known on what happens at the critical speed $n_{k} \sim e^{c \sqrt{k}}$ (critical behavior) and what is the probabilistic nature of $\left(\cos n_{k} x\right)$ in the strongly dependent domain. In our paper we study the critical LIL behavior of $\left(\cos n_{k} x\right)$ i.e., we investigate how classical fluctuational theorems like the law of the iterated logarithm and the Kolmogorov-Feller test turn to nonclassical laws in the immediate neighborhood of $n_{k} \sim e^{c \sqrt{k}}$.
\end{abstract}

\section{INTRODUCTION}

The purpose of this paper is to study the probabilistic behavior of lacunary trigonometric series. Specifically, we shall give essentially optimal lacunarity conditions under which a subsequence of the trigonometric system satisfies the law of the iterated logarithm and some of its refinements, e.g., the KolmogorovFeller upper-lower class test. We shall also investigate critical phenomena related to the LIL, i.e. study the surprising properties of lacunary trigonometric series in the immediate neighborhood of the gap condition where the law of the iterated logarithm and the Kolmogorov-Feller test break down.

It is well known that for rapidly increasing $\left(n_{k}\right)$ the sequences $\left(\sin n_{k} x\right)_{k=1}^{\infty}$, $\left(\cos n_{k} x\right)_{k=1}^{\infty}$ behave like sequences of independent random variables. ${ }^{1}$ For example, if $\left(n_{k}\right)$ is a sequence of positive integers satisfying

$$
n_{k+1} / n_{k} \geq q>1 \quad(k=1,2, \ldots)
$$

then by classical results of Salem-Zygmund [14] and Erdös-Gál [6] we have

$$
\lim _{N \rightarrow \infty} \frac{1}{2 \pi} \lambda\left(0 \leq x \leq 2 \pi: \sum_{k \leq N} \cos n_{k} x<t \sqrt{N / 2}\right)=\boldsymbol{\Phi}(t)
$$

Received by the editors February 20, 1991.

1991 Mathematics Subject Classification. Primary 42A55, 60F15.

Key words and phrases. Lacunary trigonometric series, weak and strong dependence, law of the iterated logarithm, upper-lower class tests.

Research supported by Hungarian National Foundation for Scientific Research, Grant No. 1905.

${ }^{1}$ Throughout this paper, the probability space for the trigonometric system will be $(0,2 \pi)$, equipped with the Borel $\sigma$-field $\mathscr{B}$ and normalized Lebesgue measure $(2 \pi)^{-1} \lambda$. 
and

$$
\varlimsup_{N \rightarrow \infty}(N \log \log N)^{-1 / 2} \sum_{k \leq N} \cos n_{k} x=1 \quad \text { a.e. }^{2}
$$

where $\Phi(t)=(2 \pi)^{-1 / 2} \int_{-\infty}^{t} \exp \left(-u^{2} / 2\right) d u$ is the standard normal distribution function. Actually, much more than (1.2) and (1.3) is true: Philipp and Stout [12] proved that under (1.1) the partial sum process $S(t)=S(t, x)=$ $\sum_{k \leq t} \cos n_{k} x \quad(t \geq 0)$ is nearly Wiener in the sense that without changing its distribution it can be redefined on a suitable probability space together with a Wiener process $\{W(t), t \geq 0\}$ such that

$$
S(t)=W(t / 2)+O\left(t^{1 / 2-\rho}\right) \quad \text { a.s. as } t \rightarrow \infty
$$

for some constant $\rho>0$. The approximation (1.4) implies not only the central limit theorem (1.2) and the law of the iterated logarithm (1.3) but it extends a large class of limit theorems of independent r.v.'s to the sequence $\left(\cos n_{k} x\right)$. For example, (1.4) implies easily that $\left(\cos n_{k} x\right)$ obeys the Kolmogorov-Feller test, i.e., for any positive nondecreasing sequence $\varphi_{N}$ the inequality

$$
\sum_{k \leq N} \cos n_{k} x>\sqrt{N / 2} \varphi_{N}
$$

holds a.e. for finitely or infinitely many $N$ according as the series

$$
\sum_{N \geq 1} \frac{\varphi_{N}}{N} \exp \left(-\frac{1}{2} \varphi_{N}^{2}\right)
$$

converges or diverges. Since the same test is valid for the Wiener process, we see that under (1.1) the partial sum growth of $\left(\cos n_{k} x\right)$ is exactly the same as that of i.i.d.r.v.'s.

All the results formulated above concern the case of the Hadamard gap condition (1.1) and in fact most known probabilistic results for $\left(\cos n_{k} x\right)$ in the literature assume (1.1). (For a survey of the existing results before 1966 see [10]; for modern results see e.g. [11].) Erdös was the first to note that the near independent behavior of $\left(\cos n_{k} x\right)$ remains valid for a large class of sequences $\left(n_{k}\right)$ growing slower than exponential; in fact he proved the following result:

Theorem A (Erdös 1962). Let $\left(n_{k}\right)$ be a sequence of positive integers satisfying

$$
n_{k+1} / n_{k} \geq 1+c_{k} / \sqrt{k}, \quad c_{k} \rightarrow \infty \text {. }
$$

Then $\left(\cos n_{k} x\right)$ satisfies the CLT i.e. (1.2) holds. On the other hand, for every $c>0$ there exists a sequence $\left(n_{k}\right)$ of integers satisfying

$$
n_{k+1} / n_{k} \geq 1+c / \sqrt{k} \quad\left(k \geq k_{0}\right)
$$

such that the CLT (1.2) is false.

To understand the meaning of (1.5) let us say, given positive numerical sequences $\left(a_{N}\right),\left(b_{N}\right)$, that $a_{N} \succ b_{N}$ if $a_{N+1} / a_{N} \geq b_{N+1} / b_{N}$ for $N \geq N_{0}$. Then Erdős' theorem shows that $\left(\cos n_{k} x\right)$ satisfies the CLT if $n_{k} \succ e^{c_{k} \sqrt{k}}$ for some $c_{k} \uparrow \infty$ and for $n_{k} \succ e^{c \sqrt{k}}$ the result breaks down. An analogous, but slightly less precise result for the LIL was found by Takahashi:

\footnotetext{
${ }^{2}$ Relations (1.2), (1.3) remain valid also for sine series $\sum \sin n_{k} x$ and more generally, for $\sum \cos \left(n_{k} x+\varphi_{k}\right)$ where $\varphi_{k}$ are arbitrary real numbers. To simplify the formulas, however, in our paper we shall deal only with pure cosine series.
} 
Theorem B (Takahashi 1972, 1975). Let $\left(n_{k}\right)$ be a sequence of positive integers satisfying

$$
n_{k} \succ e^{k^{\alpha}}, \quad \alpha>1 / 2 .
$$

Then $\left(\cos n_{k} x\right)$ obeys the LIL (1.3). On the other hand, there exists a sequence $\left(n_{k}\right)$ of integers satisfying (1.6) with $\alpha=1 / 2$ such that the LIL (1.3) is false. ${ }^{3}$

Theorems A and B show that at the speed $n_{k} \sim e^{c \sqrt{k}}$ the probabilistic behavior of $\left(\cos n_{k} x\right)$ undergoes a fundamental change: from almost independent the sequence turns to strongly dependent. Due to a series of remarkable papers by Takahashi (see [15-20]) the behavior of $\left(\cos n_{k} x\right)$ on the near independent side of $e^{c \sqrt{k}}$ is fairly well known; on the other hand, practically nothing is known in the strongly dependent domain. In a recent paper [3] we constructed the first class of nongaussian limit distributions of normed sums

$$
\frac{1}{a_{N}} \sum_{k \leq N} \cos n_{k} x-b_{N}
$$

in the strongly dependent case; no complete characterization of the class of limit laws of such sums is known (or seems to be easy). It is not known, either, what asymptotic result replaces the law of the iterated logarithm (1.3) in the strongly dependent domain.

The purpose of this paper is to study the LIL behavior of $\left(\cos n_{k} x\right)$ in the 'critical zone' i.e. in the immediate neighborhood of the critical speed $n_{k} \sim$ $e^{c \sqrt{k}}$. In view of the strong relation between the central limit theorem and the law of the iterated logarithm, it is natural to expect that the gap condition (1.5) implies also the law of the iterated $\operatorname{logarithm}(1.3)$ for $\left(\cos n_{k} x\right)$. Surprisingly, however, this is not the case: in [4] we constructed a sequence $\left(n_{k}\right)$ of integers satisfying (1.5) with a very slowly increasing $\left(c_{k}\right)$ such that $(1.3)$ is false. On the other hand, Theorem B above shows that (1.5) with $c_{k} \geq k^{\varepsilon} \quad(\varepsilon>0)$ implies the LIL (1.3) and in [20] it is proved that (1.5) with $c_{k} \geq k^{\varepsilon}$ implies also a version of the strong approximation theorem (1.4). These remarks show that even though the Erdös gap condition (1.5) implies the CLT (1.2) for any $c_{k} \rightarrow \infty$, the partial sum behavior of $\left(\cos n_{k} x\right)$ follows the independent pattern only if $c_{k}$ has a certain minimal speed and for very slowly increasing $c_{k}$ (i.e. near the critical speed $\left.e^{c \sqrt{k}}\right)$ the independent behavior of $\left(\cos n_{k} x\right)$ breaks down, e.g. the ordinary LIL (1.3) becomes false. The main result of our paper will show that the change from independent to strongly dependent LIL behavior of $\left(\cos n_{k} x\right)$ takes place at the speed

$$
n_{k} \sim e^{\sqrt{k}(\log \log k)^{a}} .
$$

In fact, as $\alpha$ changes, the LIL behavior of $\left(\cos n_{k} x\right)$ goes through a variety of types from "very good" to "very bad". For $\alpha$ large, the LIL behavior of $\left(\cos n_{k} x\right)$ is classical: it satisfies not only the ordinary LIL (1.3) but also the Kolmogorov-Feller test and even a slightly weaker form of the a.s. invariance principle (1.4), namely

$$
S(t)=W(t / 2)+O\left(t^{1 / 2}(\log \log t)^{-\beta}\right) \text { a.s. }
$$

\footnotetext{
${ }^{3}$ The second half of the theorem is implicit in Erdös' example in Theorem A.
} 
where $\beta=\beta(\alpha) \rightarrow \infty$ as $\alpha \rightarrow \infty$. For $\alpha$ small, $\left(\cos n_{k} x\right)$ fails even the ordinary LIL (1.3) and in fact the cluster set of

$$
\left\{(N \log \log N)^{-1 / 2} \sum_{k \leq N} \cos n_{k} x, N \geq 1\right\}
$$

can be asymmetric around zero and contain points with absolute value $>1$. For an intermediate range of $\alpha$ 's the LIL behavior of $\left(\cos n_{k} x\right)$ is "transitional": it satisfies the ordinary LIL (1.3) but fails the Kolmogorov-Feller test and the upper-lower class behavior of $\left(\cos n_{k} x\right)$ is described by an asymmetric test whose form is different from the Kolmogorov-Feller test and becomes, as $\alpha$ decreases, gradually more and more complicated. At $\alpha=1 / 2$ the test blows up and even the ordinary LIL breaks down.

We now formulate our results in detail.

Theorem 1. Let $\left(n_{k}\right)$ be a sequence of positive integers satisfying

$$
n_{k} \succ e^{\sqrt{k}(\log \log k)^{\alpha}}
$$

for some $\alpha>5 / 2$. Then $\left(\cos n_{k} x\right)$ satisfies the Kolmogorov-Feller test i.e. setting $S_{N}=\sum_{k \leq N} \cos n_{k} x$ we have for any positive nondecreasing function $\varphi_{N}$

$$
P\left\{S_{N}>\sqrt{N / 2} \varphi_{N} \text { i.o. }\right\}=0 \text { or } 1,{ }^{4}
$$

according as

$$
\sum_{N \geq 1} \frac{\varphi_{N}}{N} \exp \left(-\frac{1}{2} \varphi_{N}^{2}\right)<+\infty \text { or }=+\infty .
$$

Theorem 2. For any $1 / 2 \leq \alpha<3 / 2$ and all sufficiently small $A>0$ there exists a sequence $\left(n_{k}\right)$ of positive integers satisfying

$$
n_{k} \succ e^{\sqrt{k / 8 A}(\log \log k)^{\alpha}}
$$

such that the Kolmogorov-Feller test (1.10)-(1.11) fails for the partial sums $S_{N}=\sum_{k \leq N} \cos n_{k} x$. More precisely, given any positive nondecreasing sequence $\varphi_{N}$ satisfying

$$
2^{-1}(\log \log N)^{1 / 2} \leq \varphi_{N} \leq 2(\log \log N)^{1 / 2}
$$

the alternative in (1.10) holds according as

$$
\sum_{N \geq 1} \frac{\varphi_{N}}{N} \exp \left\{-\frac{1}{2} \sum_{\nu=0}^{\infty} \frac{a_{N, \nu}}{(\log \log N)^{\alpha \nu}} \varphi_{N}^{\nu+2}\right\}<+\infty \quad \text { or }=+\infty .
$$

Here $a_{N, \nu}(\nu=0,1, \ldots)$ are explicitly calculable numbers with

$$
\begin{gathered}
a_{N, 0}=1, \quad a_{N, 1}=\frac{\sqrt{A}}{3}, \quad a_{N, 2}=-\frac{5 A}{36}, \\
\left|a_{N, \nu}\right| \leq \frac{(48 \sqrt{A})^{\nu}}{7}, \quad N \geq 1, \quad \nu \geq 1 .
\end{gathered}
$$

\footnotetext{
${ }^{4}$ Whenever they concern the trigonometric system, the symbols $P$ and $E$ mean normalized Lebesgue measure and integral in $(0,2 \pi)$, respectively. (Cf. footnote 1.) The symbol "i.o." stands for "infinitely often".
} 
Theorem 3. Let $\left(n_{k}\right)$ be a sequence of positive integers satisfying (1.9) for $\alpha>1 / 2$. Then $\left(\cos n_{k} x\right)$ satisfies the upper half of the law of the iterated logarithm, i.e.

$$
\varlimsup_{N \rightarrow \infty}(N \log \log N)^{-1 / 2}\left|\sum_{k \leq N} \cos n_{k} x\right| \leq 1 \text { a.e. }
$$

On the other hand, there is a sequence $\left(n_{k}\right)$ satisfying (1.9) with $\alpha=1 / 2$ such that (1.3a) is false.

There is a gap between the constants $5 / 2$ and $3 / 2$ in Theorems 1 and 2 and thus the upper-!ower class behavior of $\left(\cos n_{k} x\right)$ remains open if (1.9) holds with $3 / 2 \leq \alpha \leq 5 / 2$. We believe that the example of Theorem 2 is best possible i.e. Theorem 1 holds actually for $\alpha \geq 3 / 2$. (See in this respect the remarks at the end of this section.) It seems also likely that Theorem 3 remains valid with $\leq 1$ in $(1.3 \mathrm{a})$ replaced by $=1$.

To understand the meaning of (1.14) we mention a few special cases.

1. Assume $1 \leq \alpha<3 / 2$. Then (1.14) reduces to

$$
\sum_{N \geq 1} \frac{\varphi_{N}}{N} \exp \left\{-\frac{1}{2} \varphi_{N}^{2}-\frac{\sqrt{A}}{6} \frac{\varphi_{N}^{3}}{(\log \log N)^{\alpha}}\right\}<+\infty \quad \text { or }=+\infty \text {. }
$$

2. Assume $5 / 6 \leq \alpha<1$. Then (1.14) reduces to

$$
\begin{gathered}
\sum_{N \geq 1} \frac{\varphi_{N}}{N} \exp \left\{-\frac{1}{2} \varphi_{N}^{2}-\frac{\sqrt{A}}{6} \frac{\varphi_{N}^{3}}{(\log \log N)^{\alpha}}+\frac{5 A}{72} \frac{\varphi_{N}^{4}}{(\log \log N)^{2 \alpha}}\right\} \\
<+\infty \text { or }=+\infty .
\end{gathered}
$$

Generally, let $c_{k}=(k+2) / 2 k, k=1,2, \ldots$ Then $c_{1}=3 / 2, c_{2}=1$, $c_{3}=5 / 6, \ldots, c_{1}>c_{2}>\cdots$ and $\lim _{k \rightarrow \infty} c_{k}=1 / 2$. Now if $\alpha \in\left[c_{k+1}, c_{k}\right)$ then (1.14) reduces to

$$
\begin{aligned}
\sum_{N \geq 1} \frac{\varphi_{N}}{N} \exp \{- & \frac{1}{2} \varphi_{N}^{2}-\frac{1}{2} a_{N, 1} \frac{\varphi_{N}^{3}}{(\log \log N)^{\alpha}} \\
& \left.-\cdots-\frac{1}{2} a_{N, k} \frac{\varphi_{N}^{k+2}}{(\log \log N)^{k \alpha}}\right\}<+\infty \text { or }=+\infty .
\end{aligned}
$$

In other words, if $\alpha$ decreases from $3 / 2$ to $1 / 2$ then the test $(1.10)-(1.14)$ becomes gradually more and more complicated: passing each value $c_{k}$, one new term appears in the exponent in (1.18). For $\alpha>1 / 2$ the first term in the exponent in (1.14) dominates (for $\varphi_{N}$ satisfying (1.13)) and thus in this case $\left(\cos n_{k} x\right)$ satisfies the ordinary LIL (1.3). For $\alpha=1 / 2$, on the other hand, all terms in the exponent in (1.14) have the same order of magnitude as the first term $-\varphi_{N}^{2} / 2$ and this leads to a change in (1.3). In fact in this case the test (1.10)-(1.14) implies

$$
\varlimsup_{N \rightarrow \infty}(N \log \log N)^{-1 / 2} \sum_{k \leq N} \cos n_{k} x=1-\frac{1}{6} \sqrt{2 A}+O\left(A^{2 / 3}\right) \text { a.e. }
$$

i.e. for sufficiently small $A$ the $\varlimsup_{\lim }$ in (1.3) is $<1$. 
To get further comparisons between the test (1.10)-(1.14) and the Kolmogorov-Feller test (1.10)-(1.11) we recall the well-known fact that if the Kolmogorov-Feller test holds then

$$
\varphi_{N}=\left(2 \log _{2} N+3 \log _{3} N+2 \log _{4} N+\cdots+2 \log _{l-1} N+(2+\varepsilon) \log _{l} N\right)^{1 / 2}
$$

belongs to the upper or lower class (i.e. the first or second alternative in (1.10) holds) according as $\varepsilon>0$ or $\varepsilon \leq 0$. (Here $\log _{l}$ denotes the $l$ times iterated logarithm.) Under the test (1.10)-(1.14) this will change, namely we have to insert some new terms between $2 \log _{2} N$ and $3 \log _{3} N$ in (1.20) as the following corollary shows. Set

$$
\rho_{\varepsilon}(N)=3 \log _{3} N+2 \log _{4} N+\cdots+2 \log _{l-1} N+(2+\varepsilon) \log _{l} N .
$$

Then we have

Corollary. Let $1 / 2<\alpha<3 / 2$, then

$$
\varphi_{N}=\left(2 \log \log N+\sum_{j=0}^{k(\alpha)} b_{N, j}(\log \log N)^{3 / 2-\alpha-d j}+\rho_{\varepsilon}(N)\right)^{1 / 2}
$$

belongs to the upper or lower class with respect to $\left(\cos n_{k} x\right)$ according as $\varepsilon>0$ or $\varepsilon \leq 0$. Here $k=k(\alpha)$ is the integer defined by $\alpha \in\left[c_{k+2}, c_{k+1}\right), d=\alpha-1 / 2>0$ and the $b_{N, j}$ are explicitly calculable numbers (actually polynomials of the $a_{N, j}$ in (1.14)). In particular $b_{N, 0}=-2 \sqrt{2 A} / 3, b_{N, 1}=11 A / 9$.

Again we mention a few special cases.

1. Let $1 / 2<\alpha<3 / 2$. Then

$$
\varphi_{N}=\left(2 \log \log N-c(\log \log N)^{3 / 2-\alpha}\right)^{1 / 2}
$$

belongs to the upper or lower class according as $c<2 \sqrt{2 A} / 3$ or $c \geq 2 \sqrt{2 A} / 3$.

2. Let $1 \leq \alpha<3 / 2$. Then

$$
\varphi_{N}=\left(2 \log \log N-\frac{2 \sqrt{2 A}}{3}(\log \log N)^{3 / 2-\alpha}+\rho_{\varepsilon}(N)\right)^{1 / 2}
$$

belongs to the upper or lower class according as $\varepsilon>0$ or $\varepsilon \leq 0$.

3. Let $5 / 6 \leq \alpha<1$. Then

$$
\varphi_{N}=\left(2 \log \log N-\frac{2 \sqrt{2 A}}{3}(\log \log N)^{3 / 2-\alpha}+\frac{11 A}{9}(\log \log N)^{2-2 \alpha}+\rho_{\varepsilon}(N)\right)^{1 / 2}
$$

belongs to the upper or lower class according as $\varepsilon>0$ or $\varepsilon \leq 0$.

A remarkable additional property of the sequence $\left(n_{k}\right)$ constructed in Theorem 2 is that the sequences $\left(\cos n_{k} x\right)$ and $\left(-\cos n_{k} x\right)$ have different upperlower class behavior. Indeed, the proof of Theorem 2 shows that for any positive nondecreasing sequence $\varphi_{N}$ satisfying (1.13) we have

$$
P\left\{-S_{N}>\sqrt{N / 2} \varphi_{N} \text { i.o. }\right\}=0 \text { or } 1
$$


according as

$$
\sum_{N \geq 1} \frac{\varphi_{N}}{N} \exp \left\{-\frac{1}{2} \sum_{\nu=0}^{\infty} \frac{b_{N, \nu}}{(\log \log N)^{\alpha \nu}} \varphi_{N}^{\nu+2}\right\}<+\infty \quad \text { or }=+\infty .
$$

Here $b_{N, \nu}(\nu \geq 0)$ are explicitly calculable numbers satisfying the same inequalities

$$
\left|b_{N, \nu}\right| \leq \frac{(48 \sqrt{A})^{\nu}}{7}, \quad N \geq 1, \nu \geq 1
$$

as we have for the $a_{N, \nu}$ but the sequences $\left(a_{N, \nu}\right)_{\nu \geq 0}$ and $\left(b_{N, \nu}\right)_{\nu \geq 0}$ are different. In particular,

$$
b_{N, 0}=1, \quad b_{N, 1}=-\frac{\sqrt{A}}{3}, \quad b_{N, 2}=-\frac{5 A}{36} .
$$

An immediate consequence of this asymmetry is that for $\alpha=1 / 2$ the cluster set of $(N \log \log N)^{-1 / 2} \sum_{k \leq N} \cos n_{k} x$ is not symmetric around zero; in fact we have

$$
\varliminf_{N \rightarrow \infty}(N \log \log N)^{-1 / 2} \sum_{k \leq N} \cos n_{k} x=-1-\frac{1}{6} \sqrt{2 A}+O\left(A^{2 / 3}\right) \text { a.e. }
$$

Hence

$$
\varlimsup_{N \rightarrow \infty}(N \log \log N)^{-1 / 2}\left|\sum_{k \leq N} \cos n_{k} x\right|>1 \quad \text { a.e. }
$$

if $A$ is small enough.

There is a remarkable similarity between the change of the upper-lower class behavior of $\left(\cos n_{k} x\right)$ described by Theorem 2 and the change of the upperlower class behavior of independent sequences $\left(X_{n}\right)$ under the condition

$$
\begin{gathered}
E X_{n}=0, \quad E X_{n}^{2}<+\infty, \quad s_{n}^{2}=\sum_{k \leq n} E X_{k}^{2} \rightarrow+\infty, \\
\left|X_{n}\right| \leq K \frac{s_{n}}{\left(\log \log s_{n}\right)^{\beta}} \quad(\beta \geq 1 / 2)
\end{gathered}
$$

as $\beta$ changes. Feller [8] showed that under (1.23) the upper-lower class behavior of $\left(X_{n}\right)$ is described by an integral test whose form is getting more and more complicated as $\beta$ decreases; as $\beta$ approaches $1 / 2$, successively higher and higher moments of the $\left(X_{n}\right)$ enter the test. Formal analogy with Theorem 2 leads to the conjecture that $\alpha=3 / 2$ is the critical exponent for the Kolmogorov-Feller test for $\left(\cos n_{k} x\right)$ i.e. Theorem 1 holds actually for $\alpha \geq 3 / 2$. It is also natural to expect that under conditions similar to those of Theorem $1\left(\cos n_{k} x\right)$ obeys Chung's test for partial maxima, i.e. setting $S_{N}^{*}=\max _{1 \leq M \leq N}\left|\sum_{k \leq M} \cos n_{k} x\right|$ we have for any positive increasing sequence $\psi_{N}$

$$
P\left\{S_{N}^{*}<\sqrt{N / 2} \psi_{N}^{-1} \text { i.o. }\right\}=0 \text { or } 1
$$

according as

$$
\sum_{N \geq 1} \frac{\psi_{N}^{2}}{N} \exp \left\{-\frac{8 \psi_{N}^{2}}{\pi^{2}}\right\}<+\infty \text { or }=+\infty
$$


The method of the proof of Theorem 1 shows (see the remark at the end of $\S 3)$ that $\left(\cos n_{k} x\right)$ satisfies the test (1.24)-(1.25) if (1.9) holds with $\alpha>7 / 2$; whether $\alpha>5 / 2$ (or even $\alpha \geq 3 / 2$ ) is also sufficient remains open. The proof of Theorem 1 also shows that under (1.9) with $\alpha>3 / 2\left(\cos n_{k} x\right)$ satisfies the a.s. invariance principle (1.4) in the slightly weaker form

$$
S(t)=W(t / 2)+O\left(t^{1 / 2}(\log \log t)^{(5-2 \alpha+\varepsilon) / 4}\right) \quad \text { a.s. }
$$

for any $\varepsilon>0$. It should be noted that the proof of Theorem 1 will not proceed via the a.s. approximation (1.26). In fact, while this traditional approach would work, it would mean a loss of precision: (1.26) implies the Kolmogorov-Feller test (1.10)-(1.11) only for $\alpha>7 / 2$ i.e. under stronger assumptions than we assumed in Theorem 1. Hence, while in the standard theory of weakly dependent r.v.'s (see e.g. [12]) the a.s. invariance principle, the Kolmogorov-Feller and the Chung tests are obtained simultaneously, under the same conditions, in the very delicate trigonometric situation around the critical speed $e^{c \sqrt{k}}$ the difference between the above limit theorems becomes essential.

It is worth noting that condition (1.13) in Theorem 2 cannot be omitted completely since (1.16) guarantees the convergence of

$$
\sum_{\nu=0}^{\infty} a_{N, \nu}(\log \log N)^{-\alpha \nu} x^{\nu+2}
$$

only for $|x|<(48 \sqrt{A})^{-1}(\log \log N)^{\alpha}$ and thus for too large $\varphi_{N}$ the series in the exponent of (1.14) may become divergent. However, a standard argument shows (see [9, Lemma 2] and [8, p. 398]) that for any positive nondecreasing $\varphi_{N}$ the probability in (1.10) does not change if we replace $\varphi_{N}$ by the nondecreasing sequence $\psi_{N}$ defined by

$$
\psi_{N}= \begin{cases}2^{-1}(\log \log N)^{1 / 2} & \text { if } \varphi_{N} \leq 2^{-1}(\log \log N)^{1 / 2} \\ 2(\log \log N)^{1 / 2} & \text { if } \varphi_{N} \geq 2(\log \log N)^{1 / 2} \\ \varphi_{N} & \text { otherwise. }\end{cases}
$$

Thus Theorem 2 permits us to decide, for any nondecreasing $\varphi_{N}$, if the probability in (1.10) is 0 or 1 .

In conclusion we note that Theorem 2 states only that the LIL behavior of $\left(\cos n_{k} x\right)$ is bad for some sequences $\left(n_{k}\right)$ satisfying (1.9), $1 / 2 \leq \alpha<3 / 2$ but not that all sequences $\left(n_{k}\right)$ with the same speed have bad LIL behavior. As we shall prove at the end of $\S 4$, given any sequence $\left(n_{k}\right)$ satisfying (1.12) for some $A>0, \alpha>0$, there exists a sequence $\left(m_{k}\right)$ such that $\left|m_{k}-n_{k}\right| \leq$ const $\cdot k^{3}$ (and thus $\left.m_{k} \sim n_{k}\right)$ such that $\left(\cos m_{k} x\right)$ satisfies the Kolmogorov-Feller test (1.10)-(1.11). This remark also shows that near the critical speed $n_{k} \sim e^{c \sqrt{k}}$ the LIL behavior of $\left(\cos n_{k} x\right)$ becomes very unstable: small relative changes in $\left(n_{k}\right)$ lead to essential changes in the behavior of $\left(\cos n_{k} x\right)$.

The proof of Theorems 1 and 2 will be given in $\S \S 2-3$ and in $\S 4$, respectively. As we noted above, for $\alpha=1 / 2$ the sequence $\left(\cos n_{k} x\right)$ constructed in Theorem 2 fails the upper LIL (1.3a) (see the remarks on the asymmetric behavior of $\left.\left(\cos n_{k} x\right)\right)$ and thus the second half of Theorem 3 is also contained in the proof of Theorem 2. The proof of the first half of Theorem 3 requires combinatorial tools similar to those used for Theorem 1 but the details are considerably more 
complicated. Hence to keep our paper at a reasonable length, we will give the proof in a subsequent paper.

\section{SOME LEMMAS}

The crucial step of the proof of Theorem 1 is Lemma 2.1 below giving a fairly sharp estimate for the number of solutions of a certain diophantine equation.

Lemma 2.1. Let $\left\{n_{j}, M+1 \leq j \leq M+N\right\}$ be a finite sequence of positive integers such that

$$
n_{j+1} / n_{j} \geq 1+c / \sqrt{j}, \quad M+1 \leq j \leq M+N-1 .
$$

Let $p \geq 2$ be an integer and assume that

$$
c \sqrt{N p} \leq \sqrt{M+N} \leq c N / 48 \text {. }
$$

Let finally $\varepsilon_{1}, \ldots, \varepsilon_{p}$ be a sequence of \pm 1 's and $d$ an arbitrary integer. Then the number of solutions of the equation

$$
\begin{aligned}
\varepsilon_{1} n_{i_{1}}+\varepsilon_{2} n_{i_{2}}+\cdots+ & \varepsilon_{p} n_{i_{p}}=d \\
& \left(M+N \geq i_{1} \geq i_{2} \geq \cdots \geq i_{p} \geq M+1\right)
\end{aligned}
$$

is at most

$$
2(576 \log p)^{p} N(\sqrt{M+N} / c)^{p-2} .
$$

For the proof we need some preparatory lemmas.

Lemma 2.2. Let $\left\{n_{j}, M+1 \leq j \leq M+N\right\}$ satisfy (2.1) and assume that $M+N \geq c^{2}$. Then for any $0<a<b$ the interval $[a, b]$ contains at most

$$
2 c^{-1} \sqrt{M+N} \log b / a+1
$$

terms of the sequence $\left\{n_{j}, M+1 \leq j \leq M+N\right\}$.

Proof. Let $n_{q}$ and $n_{r}$ be the smallest and largest among the $n_{j}$ 's $(M+1 \leq j \leq$ $M+N)$ in the interval $[a, b]$. Then $n_{r} / n_{q} \leq b / a$; on the other hand by $(2.1)$ we have

$$
\frac{n_{r}}{n_{q}} \geq \prod_{j=q}^{r-1}\left(1+\frac{c}{\sqrt{j}}\right) \geq\left(1+\frac{c}{\sqrt{M+N}}\right)^{r-q} \geq \exp \left(\frac{c}{2 \sqrt{M+N}}(r-q)\right)
$$

using the fact that $1+x \geq e^{x / 2}$ for $0 \leq x \leq 1$. The two estimates for $n_{r} / n_{q}$ imply

$$
\exp \left(\frac{c}{2 \sqrt{M+N}}(r-q)\right) \leq b / a
$$

whence $r-q+1 \leq 2 c^{-1} \sqrt{M+N} \log b / a+1$, as stated.

To simplify the writing, in the sequel we shall use the symbol $a \asymp 2^{j}$ to denote $2^{j} \leq a<2^{j+1}$.

Lemma 2.3. Assume $M+N \geq c^{2}$ and consider those solutions of (2.3) where $n_{i_{\nu}} / n_{i_{\nu+1}} \asymp 2^{j_{\nu}}(\nu=1, \ldots, p-1)$ where $j_{1}, \ldots, j_{p-1}$ are fixed nonnegative integers. Then, given $n_{i_{1}}, \ldots, n_{i_{k-1}}(2 \leq k \leq p-1)$, the number of choices for $n_{i_{k}}$ is at most

$$
\begin{array}{ll}
48 c^{-1} \sqrt{M+N} & \text { if } p 2^{-j_{k}} \geq 1 / 8, \\
48 c^{-1} \sqrt{M+N} \cdot p 2^{-j_{k}} & \text { if } c /(32 \sqrt{M+N}) \leq p 2^{-j_{k}}<1 / 8, \\
1 & \text { if } p 2^{-j_{k}}<c /(32 \sqrt{M+N}) .
\end{array}
$$


The results remain true also for $k=1$ (i.e., for the number of choices of $n_{i_{1}}$ ) except that in this case $48 c^{-1} \sqrt{M+N}$ in the first line of (2.5) should be replaced by $N$.

Proof. Assume $k \geq 2$; the argument for $k=1$ is identical (the first alternative in (2.5) is trivial for $k=1)$. By $n_{i_{k-1}} / n_{i_{k}} \asymp 2^{j_{k-1}}$ we have $n_{i_{k}} \in$ $\left[2^{-j_{k-1}-1} n_{i_{k-1}}, 2^{-j_{k-1}} n_{i_{k-1}}\right]$. Hence using Lemma 2.2 it follows that given $n_{i_{1}}$, $\ldots, n_{i_{k-1}}$, for $n_{i_{k}}$ we have at most $2 c^{-1} \sqrt{M+N} \log 2+1 \leq 3 c^{-1} \sqrt{M+N}$ choices, no matter which assumption on $p 2^{-j_{k}}$ in (2.5) holds. Thus the estimate in the first line of (2.5) is proved. Assume now $p 2^{-j_{k}}<1 / 8$. Let $n_{i_{1}}, \ldots, n_{i_{k-1}}$ be given and let $\varepsilon_{1} n_{i_{1}}+\cdots+\varepsilon_{k-1} n_{i_{k-1}}=A$. By $n_{i_{k}} / n_{i_{k+1}} \asymp 2^{j_{k}}$ it follows that the numbers $n_{i_{k+1}}, n_{i_{k+2}}, \ldots, n_{i_{p}}$ are all $\leq n_{i_{k}} 2^{-j_{k}}$ and thus $\left|\varepsilon_{k+1} n_{i_{k+1}}+\cdots+\varepsilon_{p} n_{i_{p}}\right| \leq p n_{i_{k}} 2^{-j_{k}}$. Hence (2.3) yields

$$
A+\varepsilon_{k} n_{i_{k}}\left(1+\theta p 2^{-j_{k}}\right)=d, \quad|\theta| \leq 1 .
$$

Thus, setting $B=(d-A) / \varepsilon_{k}$ and using $p 2^{-j_{k}}<1 / 8$ and the fact that for $|x| \leq 1 / 2$ we have $(1+x)^{-1}=1+\lambda x$ with $|\lambda| \leq 2$, we get

$$
n_{i_{k}}=B\left(1+\theta p 2^{-j_{k}}\right)^{-1}=B\left(1+\theta^{\prime} p 2^{-j_{k}}\right), \quad\left|\theta^{\prime}\right| \leq 2 .
$$

Here $B \neq 0$ since $n_{i_{k}} \neq 0$. Thus using Lemma 2.2 it follows that there are at most

$$
\begin{aligned}
2 c^{-1} \sqrt{M+N} \log \frac{1+2 p 2^{-j_{k}}}{1-2 p 2^{-j_{k}}}+1 & \leq 2 c^{-1} \sqrt{M+N} \log \left(1+6 p 2^{-j_{k}}\right)+1 \\
& \leq 12 c^{-1} \sqrt{M+N} p 2^{-j_{k}}+1
\end{aligned}
$$

choices for $n_{i_{k}}$. It remains now to observe that the last expression in (2.7) is bounded by $48 c^{-1} \sqrt{M+N} p 2^{-j_{k}}$ or $3 / 2$ according as $p 2^{-j_{k}}$ satisfies the inequality in the second or third line of $(2.5)$.

Remark 2.4. In Lemma 2.3 we estimated the number of choices for $n_{i_{k}}$ in the diophantine equation (2.3) provided $n_{i_{1}}, \ldots, n_{i_{k-1}}$ are given and provided we consider only those solutions of $(2.3)$ such that $n_{i_{\nu}} / n_{i_{\nu+1}} \asymp 2^{j_{\nu}} \quad(1 \leq \nu \leq p-1)$ where $j_{1}, \ldots, j_{p-1}$ are fixed nonnegative integers. Note, however, that for the estimate in the second and third line of $(2.5)$ we used only the fact that $j_{k}$ is fixed (for the estimate in the first line we need also that $j_{k-1}$ is fixed). Observe also that in the third line of (2.5) the number of choices for $n_{i_{k}}$ is $\leq 1$ not only for any fixed $j_{k}$ with $p 2^{-j_{k}}<c /(32 \sqrt{M+N})$ but for all such $j_{k}$ 's combined. In fact, in the proof we saw that if $n_{i_{1}}, \ldots, n_{i_{k-1}}$ are given and $n_{i_{k}} / n_{i_{k+1}} \asymp 2^{j_{k}}$ with $p 2^{-j_{k}}<1 / 8$, then $n_{i_{k}} \in I_{k}$, where $I_{k}=\left[B\left(1-2 p 2^{-j_{k}}\right), B\left(1+2 p 2^{-j_{k}}\right)\right]$ where $B$ is a number uniquely determined by $n_{i_{1}}, \ldots, n_{i_{k-1}}$. We saw also that for $p 2^{-j_{k}}<c /(32 \sqrt{M+N})$ the interval $I_{k}$ contains at most one integer. Clearly, for increasing $j_{k}$, the intervals $I_{k}$ are shrinking and thus the union

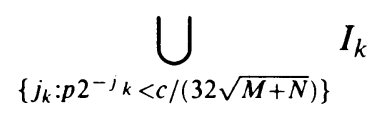

contains also at most one integer.

Proof of Lemma 2.1. To simplify the writing, we introduce some terminology. Given a solution $\left(n_{i_{1}}, \ldots, n_{i_{p}}\right)$ of $(2.3)$, the ratios $n_{i_{k}} / n_{i_{k+1}}(1 \leq k \leq p-1)$ 
will be called the gaps in this solution. For any fixed $1 \leq k \leq p-1$, the gap $n_{i_{k}} / n_{i_{k+1}}$ will be called small, medium, or large depending on whether $p 2^{-j_{k}}$ lies in the intervals $[1 / 8,+\infty),[c /(32 \sqrt{M+N}), 1 / 8)$ or $(0, c /(32 \sqrt{M+N}))$, respectively, where $n_{i_{k}} / n_{i_{k+1}} \asymp 2^{j_{k}}$. (That is, the gap $n_{i_{k}} / n_{i_{k+1}}$ is small, medium, or large according as in Lemma 2.3 the inequality in the first, second, or third line of (2.5) is valid.) Remark 2.4 shows that given $n_{i_{1}}, \ldots, n_{i_{k-1}}$ in (2.3) (but without fixing any of $\left.j_{1}, \ldots, j_{p-1}\right)$, there is at most one choice for $n_{i_{k}}$ such that the gap $n_{i_{k}} / n_{i_{k+1}}$ is large. We now proceed in steps.

1. Consider first those solutions of $(2.3)$ where $n_{i_{\nu}} / n_{i_{\nu+1}} \asymp 2^{j_{\nu}} \quad(1 \leq \nu \leq p-1)$ and all the gaps are small or medium. We separate 2 cases.

(a) The first gap $n_{i_{1}} / n_{i_{2}}$ is small. In such a solution, for $n_{i_{1}}$ there are $N$ possibilities and given $n_{i_{1}}, \ldots, n_{i_{k-1}}, 2 \leq k \leq p-1$, for $n_{i_{k}}$ there are at most $48 c^{-1} \sqrt{M+N} \psi\left(j_{k}\right)$ possibilities where the function $\psi(j)(j \geq 0)$ is defined by

$$
\psi(j)= \begin{cases}1 & \text { if } p 2^{-j} \geq 1 / 8 \\ p 2^{-j} & \text { if } p 2^{-j}<1 / 8\end{cases}
$$

Finally, given $n_{i_{1}}, \ldots, n_{i_{p-1}}$, for $n_{i_{p}}$ there is at most one possibility. Thus the number of such solutions of (2.3) is at most

$$
N(48 \sqrt{M+N} / c)^{p-2} \prod_{k=2}^{p-1} \psi\left(j_{k}\right) .
$$

(b) The gap $n_{i_{1}} / n_{i_{2}}$ is medium. In this case for $n_{i_{1}}$ there are at most $48 c^{-1} \sqrt{M+N} p 2^{-j_{1}}$ possibilities and the number of choices for the other $n_{i_{k}}$ 's can be estimated as above. Thus the number of solutions of this type is at most

$$
(48 \sqrt{M+N} / c)^{p-1} p 2^{-j_{1}} \prod_{k=2}^{p-1} \psi\left(j_{k}\right) .
$$

Adding (2.8) and (2.9) and summing for $j_{1}, \ldots, j_{p-1}$ we get an upper estimate for the number of solutions of (2.3) containing only small and medium gaps. Note that

$$
\sum_{j=0}^{\infty} \psi(j)=\sum_{p 2^{-j} \geq 1 / 8} 1+\sum_{p 2^{-j<1 / 8}} p 2^{-j} \leq 2 \log 8 p+1+1 / 4 \leq 12 \log p \quad(p \geq 2)
$$

since the last sum is a geometric series with ratio $1 / 2$ and first term $<1 / 8$. Now in case (a), the gap $n_{i_{1}} / n_{i_{2}}$ is small i.e., $p 2^{-j_{1}} \geq 1 / 8$ thus $j_{1} \leq 2 \log 8 p \leq$ $12 \log p$. Hence adding $(2.8)$ for $j_{1}, \ldots, j_{p-1}$ we get at most

$$
\begin{gathered}
N(48 \sqrt{M+N} / c)^{p-2} 12 \log p \prod_{k=2}^{p-1}\left(\sum_{j_{k}=0}^{\infty} \psi\left(j_{k}\right)\right) \\
\leq N(48 \sqrt{M+N} / c)^{p-2}(12 \log p)^{p-1} .
\end{gathered}
$$

In case (b), we have $p 2^{-j_{1}}<1 / 8$ i.e., adding (2.9) for $j_{1}, \ldots, j_{p-1}$ we get at 
most

$$
\begin{aligned}
(48 & \sqrt{M+N} / c)^{p-1} \sum_{p 2^{-j_{1}<1 / 8}} p 2^{-j_{1}} \prod_{k=2}^{p-1}\left(\sum_{j_{k}=0}^{\infty} \psi\left(j_{k}\right)\right) \\
& \leq(48 \sqrt{M+N} / c)^{p-1} \frac{1}{4}(12 \log p)^{p-2} \\
& \leq N(48 \sqrt{M+N} / c)^{p-2}(12 \log p)^{p-2},
\end{aligned}
$$

where in the last step we used the second inequality of (2.2). Thus we proved that the number of solutions of (2.3) containing no large gaps is

$$
\leq N(48 \sqrt{M+N} / c)^{p-2}(12 \log p)^{p} \quad(p \geq 2) .
$$

2. Let us consider now those solutions of (2.3) where there is exactly one large gap, say $n_{i_{s}} / n_{i_{s+1}}$. Then the $s$-tuple $\left(n_{i_{1}}, \ldots, n_{i_{s}}\right)$ and the $(p-s)$-tuple $\left(n_{i_{s+1}}, \ldots, n_{i_{p}}\right)$ contain no large gaps and by Remark 2.4 for $n_{i_{s}}$ there is at most one possibility provided the previous $n_{i_{\nu}}$ 's are given. (The same is trivially true for $\left.n_{i_{p}}.\right)$ Hence the previous argument can be applied for both $\left(n_{i_{1}}, \ldots, n_{i_{s}}\right)$ and $\left(n_{i_{s+1}}, \ldots, n_{i_{p}}\right)$ and it follows that for $\left(n_{i_{1}}, \ldots, n_{i_{s}}\right)$ we have at most

$$
N(48 \sqrt{M+N} / c)^{s-2}(12 \log p)^{s}
$$

choices and given $\left(n_{i_{1}}, \ldots, n_{i_{s}}\right)$ we have for $\left(n_{i_{s+1}}, \ldots, n_{i_{p}}\right)$ at most

$$
N(48 \sqrt{M+N} / c)^{p-s-2}(12 \log p)^{p-s}
$$

choices. Here we assumed $2 \leq s \leq p-2$ but the estimates (2.10) and (2.11) remain valid also for $s=1$ and $s=p-1$. (Indeed, if e.g. $s=1$ then the gap $n_{i_{1}} / n_{i_{2}}$ is large i.e., by Remark 2.4 there is at most one choice for $n_{i_{1}}$ while $(2.10)$ gives $(c N / 48 \sqrt{M+N}) \cdot 12 \log p$ which is greater than 1 by the second inequality of (2.2).) Since the location of the large gap $n_{i_{s}} / n_{i_{s+1}}$ can be chosen in $p-1$ different ways, it follows that the number of solutions of (2.3) containing exactly one large gap is at most

$$
N^{2}(48 \sqrt{M+N} / c)^{p-4}(12 \log p)^{p}(p-1)
$$

Similarly, the number of solutions of (2.3) containing exactly $l$ large gaps $(0 \leq l \leq p-1)$ is at most

$$
N^{l+1}(48 \sqrt{M+N} / c)^{p-2-2 l}(12 \log p)^{p}\left(\begin{array}{c}
p-1 \\
l
\end{array}\right) .
$$

Adding for $l=0,1, \ldots, p-1$ and using $\left(\begin{array}{c}p-1 \\ l\end{array}\right) \leq p^{l}$ we get that the total number of solutions of $(2.3)$ is at most

$$
\begin{aligned}
& \sum_{l=0}^{p-1} N^{l+1}(48 \sqrt{M+N} / c)^{p-2-2 l}(12 \log p)^{p} p^{l} \\
& \quad=N(48 \sqrt{M+N} / c)^{p-2}(12 \log p)^{p} \sum_{l=0}^{p-1}\left(\frac{N c^{2} p}{48^{2}(M+N)}\right)^{l} \\
& \leq 2 N(48 \sqrt{M+N} / c)^{p-2}(12 \log p)^{p}
\end{aligned}
$$

where in the last step we used that $N c^{2} p \leq M+N$ by the first inequality of (2.2). Hence Lemma 2.1 is proved. 
From Lemma 2.1 we immediately get the following moment estimate for block sums of $\left(\cos n_{k} x\right)$.

Lemma 2.5. Let $\left\{n_{j}, M+1 \leq j \leq M+N\right\}$ be a finite sequence of positive integers satisfying (2.1), further let $p \geq 2$ be an even integer and assume (2.2) holds. Then

$$
\frac{1}{2 \pi} \int_{0}^{2 \pi}\left|\sum_{k=M+1}^{M+N} \cos n_{k} x\right|^{p} d x \leq 2(576 p \log p)^{p} N(\sqrt{M+N} / c)^{p-2} .
$$

Proof. Using $\cos \alpha \cos \beta=[\cos (\alpha+\beta)+\cos (\alpha-\beta)] / 2$ it follows that the integrand in (2.12) equals $2^{-p} \sum \cos \left( \pm n_{i_{1}} \pm \cdots \pm n_{i_{p}}\right) x$, where the sum is extended for all $M+1 \leq i_{1}, \ldots, i_{p} \leq M+N$ and all possible choices of the signs \pm 1 . Since $\int_{0}^{2 \pi} \cos n x d x=0$ or $2 \pi$ according as the integer $n$ differs from zero or not we get that the left side of (2.12) equals $2^{-p}$ times the number of solutions of the equation

$$
\pm n_{i_{1}} \pm \cdots \pm n_{i_{p}}=0 \quad\left(M+1 \leq i_{1}, \ldots, i_{p} \leq M+N\right) .
$$

Fixing the signs in (2.13) and assuming $i_{1} \geq i_{2} \geq \cdots \geq i_{p}$, the number of solutions of (2.13) is bounded by the expression in (2.4). Since there are $2^{p}$ possibilities for the choice of the signs \pm 1 and $p ! \leq p^{p}$ possiblilties for the order of $i_{1}, \ldots, i_{p},(2.12)$ follows.

We complement Lemma 2.5 with the following simple estimates for the first and second moments of the trigonometric sums appearing in (2.12).

Lemma 2.6. Let $\left\{n_{j}, M+1 \leq j \leq M+N\right\}$ be a finite sequence of positive numbers satisfying (2.1) with some $c \geq 1$. Then for any $0 \leq a<b \leq 2 \pi$ we have

$$
\begin{gathered}
\int_{a}^{b}\left(\sum_{j=M+1}^{M+N} \cos n_{j} x\right) d x=O\left(N / n_{M+1}\right), \\
\int_{a}^{b}\left(\sum_{j=M+1}^{M+N} \cos n_{j} x\right)^{2} d x=\frac{1}{2} N(b-a)+O\left(N^{3} M / n_{M+1}\right),
\end{gathered}
$$

where the constants implied by the $O$ are absolute.

Proof. (2.14) follows immediately from the fact that

$$
\left|\int_{a}^{b} \cos \gamma x d x\right| \leq 2 /|\gamma| \quad(\gamma \neq 0) .
$$

Since the integrand in (2.15) equals

$$
\frac{1}{2} N+\frac{1}{2} \sum_{j=M+1}^{M+N} \cos 2 n_{j} x+\sum_{M+1 \leq \mu<\nu \leq M+N}\left[\cos \left(n_{\nu}+n_{\mu}\right) x+\cos \left(n_{\nu}-n_{\mu}\right) x\right]
$$


(2.15) follows from (2.16) and the estimate

$$
\begin{aligned}
& \sum_{M+1} \leq \mu<\nu \leq M+N \\
& \left.\leq 2 N \sum_{\nu=M+1}^{M+N-1} \frac{1}{n_{\nu}+n_{\mu}}+\frac{1}{n_{\nu}-n_{\mu}}\right) \leq \sum_{M+1 \leq \mu<\nu \leq M+N} \frac{2}{n_{\nu}-n_{\mu}} \\
& \leq 2 N \sum_{\nu=M+1}^{M+N-1} \frac{\sqrt{\nu}}{n_{\nu}} \leq \frac{2 N^{2}(M+N)}{n_{M+1}} .
\end{aligned}
$$

In the proof of Theorem 1 we shall also make use of a recent upper-lower class result for martingales, due to Einmahl and Mason [5] which we state here, for the purpose of reference, as a lemma.

Lemma 2.7. Let $\left\{X_{n}, \mathscr{F}_{n}, n \geq 1\right\}$ be a martingale difference sequence with finite second moments such that

$$
s_{n}^{2}:=\sum_{j=1}^{n} E\left(X_{j}^{2} \mid \mathscr{F}_{j-1}\right) \rightarrow+\infty \text { a.s. }
$$

and

$$
\left|X_{n}\right| \leq M s_{n} /\left(\log \log s_{n}\right)^{3 / 2} \quad \text { a.s. }
$$

for some constant $M>0$. Let $S_{n}=\sum_{j=1}^{n} X_{j}$. Then for any nondecreasing function $\varphi:(0, \infty) \rightarrow(0, \infty)$ we have

$$
P\left(S_{n}>s_{n} \varphi\left(s_{n}^{2}\right) \text { i.o. }\right)=0 \text { or } 1
$$

depending on whether

$$
\int_{1}^{\infty} \frac{\varphi(t)}{t} \exp \left(-\frac{1}{2} \varphi^{2}(t)\right) d t<+\infty \text { or }=+\infty .
$$

Moreover, if $\varphi:(0, \infty) \rightarrow(0, \infty)$ is nondecreasing and satisfies

$$
2^{-1}(\log \log t)^{1 / 2} \leq \varphi(t) \leq 2(\log \log t)^{1 / 2} \quad\left(t \geq t_{0}\right)
$$

then

$$
P\left(S_{n}>s_{n} \varphi\left(s_{n}^{2}\right) \text { i.o. }\right)=P\left(S_{n}>\hat{s}_{n} \varphi\left(\hat{s}_{n}^{2}\right) \text { i.o. }\right)
$$

for any sequence $\left\{\hat{s}_{n}, n \geq 1\right\}$ of r.v.'s such that

$$
\hat{s}_{n}=s_{n}\left(1+O\left(\left(\log \log s_{n}\right)^{-1}\right)\right) \text { a.s. }
$$

Relation (2.22), which is implicit in [5], complements the upper-lower class test $(2.19)-(2.20)$ by stating the stability of the probability in (2.19) at small perturbations of $s_{n}$. This fact will be useful in extending Lemma 2.7 to unbounded martingale difference sequences (see Corollary 2.8) and for small perturbations of martingale difference sequences appearing in the proof of Theorem 1.

Corollary 2.8. The conclusion of Lemma 2.7 remains valid if condition (2.18) of the lemma is replaced by

$$
\sum_{n \geq 1} \frac{\left(\log \log s_{n}\right)^{3}}{s_{n}^{2}} E\left(\left|X_{n}\right|^{2} I\left(\left|X_{n}\right| \geq M s_{n}\left(\log \log s_{n}\right)^{-3 / 2}\right) \mid \mathscr{F}_{n-1}\right)<+\infty \text { a.s. }
$$

Proof. This follows from Lemma 2.7 by a simple truncation procedure like e.g. in [8, pp. 399-401]. Without loss of generality it suffices to consider functions 
$\varphi(t)$ satisfying (2.21) (see [9, Lemma 2]). Assume that $\left\{X_{n}, n \geq 1\right\}$ satisfies the conditions of Lemma 2.7 with (2.18) replaced by (2.24) and put

$$
\begin{gathered}
b_{n}=M s_{n} /\left(\log \log s_{n}\right)^{3 / 2}, \\
X_{n}^{*}=X_{n} I\left(\left|X_{n}\right| \leq b_{n}\right), \quad X_{n}^{* *}=X_{n} I\left(\left|X_{n}\right|>b_{n}\right), \\
Y_{n}=X_{n}^{*}-E\left(X_{n}^{*} \mid \mathscr{F}_{n-1}\right)=X_{n}^{*}-a_{n}, \\
a_{n}=\int_{|x| \leq b_{n}} x d V_{n}^{\mathscr{F}_{n-1}}(x),
\end{gathered}
$$

here $V_{n}^{\mathscr{F}_{n-1}}$ is the conditional distribution function of $X_{n}$ given $\mathscr{F}_{n-1}$. Clearly, $\left\{Y_{n}, \mathscr{F}_{n}, n \geq 1\right\}$ is a martingale difference sequence with $\left|Y_{n}\right| \leq 2 b_{n}$; set $s_{n}^{* 2}=$ $\sum_{j=1}^{n} E\left(Y_{j}^{2} \mid \mathscr{F}_{j-1}\right)$. Following the argument in [8, pp. 399-401] with obvious modifications (the only change is that $V_{n}$ used in [8] should be replaced in our case by $V_{n}^{\mathscr{Y}_{n-1}}$ ) we get

$$
s_{n}^{* 2}=s_{n}^{2}\left(1+O\left(\left(\log \log s_{n}\right)^{-3}\right)\right) \text { a.s. }
$$

and

$$
\sum_{i=1}^{n} X_{i}=\sum_{i=1}^{n} Y_{i}+O\left(s_{n} /\left(\log \log s_{n}\right)^{3 / 2}\right) \text { a.s. }
$$

Now Lemma 2.7 applies for $\left\{Y_{n}, n \geq 1\right\}$ and thus for any nondecreasing function $\varphi:(0, \infty) \rightarrow(0, \infty)$ satisfying $(2.21)$ and any sequence $\left\{\hat{s}_{n}, n \geq 1\right\}$ of r.v.'s satisfying (2.23) we get, using the fact the convergence or divergence of the integral in (2.20) is not affected if we replace $\varphi(t)$ by $\varphi(t) \pm C / \varphi(t)$ for any constant $C>0$,

$$
\begin{aligned}
P\left(\sum_{i=1}^{n} X_{i}>\hat{s}_{n} \varphi\left(\hat{s}_{n}^{2}\right) \text { i.o. }\right) & =P\left(\sum_{i=1}^{n} Y_{i}>\hat{s}_{n} \varphi\left(\hat{s}_{n}^{2}\right)+O\left(s_{n} /\left(\log \log s_{n}\right)^{3 / 2}\right) \text { i.o. }\right) \\
& =P\left(\sum_{i=1}^{n} Y_{i}>\hat{s}_{n}\left[\varphi\left(\hat{s}_{n}^{2}\right)+O\left(\varphi\left(\hat{s}_{n}^{2}\right)^{-1}\right)\right] \text { i.o. }\right) \\
& =P\left(\sum_{i=1}^{n} Y_{i}>\hat{s}_{n} \varphi\left(\hat{s}_{n}^{2}\right) \text { i.o. }\right) \\
& =P\left(\sum_{i=1}^{n} Y_{i}>s_{n}^{*} \varphi\left(s_{n}^{* 2}\right) \text { i.o. }\right)
\end{aligned}
$$

where in the last step we used the fact that $\hat{s}_{n}=s_{n}^{*}\left(1+O\left(\left(\log \log s_{n}^{*}\right)^{-1}\right)\right)$ a.s. by (2.23) and (2.25). Hence the proof of Corollary (2.8) is completed.

Remark 2.9. In Philipp-Stout [13] and Einmahl-Mason [5] various further limit theorems for martingales under conditions similar to (2.17), (2.18) are proved. We formulate here two which we shall need in $\S 3$ to prove some supplements to Theorem 1. Let $\left\{X_{n}, \mathscr{F}_{n}, n \geq 1\right\}$ be a martingale difference sequence with finite variances satisfying (2.17) and

$$
\left|X_{n}\right| \leq M s_{n} /\left(\log \log s_{n}\right)^{\beta} \quad \text { a.s. }
$$


for some constants $M>0$ and $\beta>0$. Let $S_{n}=\sum_{i=1}^{n} X_{i}$ and $S(t)=$ $\sum_{\left\{i \geq 1: s_{i}^{2} \leq t\right\}} X_{i}$ for $t \geq 0$. In [5] it is proved that if (2.17) and (2.26) hold for some $\beta \geq 5 / 2$ then $\left\{X_{n}, n \geq 1\right\}$ satisfies the upper-lower class test corresponding to Chung's LIL, i.e., for any nondecreasing $\varphi:(0, \infty) \rightarrow(0, \infty)$ we have

$$
P\left(\max _{1 \leq j \leq n}\left|S_{j}\right|<s_{n} / \varphi\left(s_{n}^{2}\right) \text { i.o. }\right)=0 \text { or } 1
$$

depending on whether

$$
\int_{1}^{\infty} \frac{\varphi^{2}(t)}{t} \exp \left(-\frac{8 \varphi^{2}(t)}{\pi^{2}}\right) d t<+\infty \text { or }=+\infty .
$$

Whether the original conditions of Lemma 2.7 or of Corollary 2.8 imply the test (2.27)-(2.28) is still an open problem. In Philipp-Stout [13] it is proved that if (2.17) and (2.26) hold for some $\beta>1 / 2$ then without changing its distribution the process $\{S(t), t \geq 0\}$ can be redefined on a suitable probability space together with a Wiener process $\{W(t), t \geq 0\}$ such that

$$
S(t)=W(t)+O\left(t^{1 / 2}(\log \log t)^{(3-2 \beta) / 4}\right) \text { a.s. }
$$

Using the truncation method in [8, pp. 399-401] it follows that the same conclusion holds if $(2.26)$ is replaced by

$$
\sum_{n \geq 1} \frac{\left(\log \log s_{n}\right)^{2 \beta}}{s_{n}^{2}} E\left(\left|X_{n}\right|^{2} I\left(\left|X_{n}\right| \geq M s_{n}\left(\log \log s_{n}\right)^{-\beta}\right) \mid \mathscr{F}_{n-1}\right)<+\infty \quad \text { a.s. }
$$

For $\beta$ large, (2.29) implies both the Kolmogorov-Feller and Chung tests and much more; on the other hand, to get just the tests (2.19)-(2.20) and (2.27)(2.28), the direct arguments used in [5] require slightly weaker rates. (For example, (2.29) implies the Kolmogorov-Feller test (2.19)-(2.20) for $\beta \geq 5 / 2$ while in Lemma $2.7,(2.26)$ is assumed only for $\beta \geq 3 / 2$.)

To conclude this section we formulate a maximal inequality for partial sums of Fourier series which we shall need for the proof of Theorem 1.

Lemma 2.10. Let $p \geq 2$ be an even integer and let $f \in L^{p}(0,2 \pi)$ be an even function with nonnegative Fourier coefficients. Let $s_{n}(f)$ denote the nth partial sum of the Fourier series of $f$. Then

$$
\int_{0}^{2 \pi}\left(\sup _{k \geq 1}\left|s_{2^{k}}(f)\right|\right)^{p} d x \leq A^{p} \int_{0}^{2 \pi}|f|^{p} d x,
$$

where $A>1$ is an absolute constant.

Proof. We first note the well-known fact that for any $f \in L^{p}, p>1$ we have

$$
\int_{0}^{2 \pi}\left(\sup _{k \geq 1}\left|\sigma_{k}(f)\right|\right)^{p} d x \leq K \int_{0}^{2 \pi}|f|^{p} d x
$$

where $\sigma_{k}(f)$ denotes the $k$ th $(C, 1)$ mean of the Fourier series of $f$ and $K>1$ is an absolute constant. (See [21, Chapter IV, Theorem 7.8] and the remark after Theorem 7.5 concerning $A_{r}$.) Next we observe that if $p \geq 2$ is an even integer and $g=\sum_{k=0}^{\infty} b_{k} \cos k x, h=\sum_{k=0}^{\infty} c_{k} \cos k x$ are $L^{2}$-convergent sums with $0 \leq b_{k} \leq c_{k}(k=0,1, \ldots)$ then $\|g\|_{p} \leq\|h\|_{p}$ (where the $p$ norms 
can also be $+\infty)$. Clearly we can assume $\|h\|_{p}<+\infty$; let $g_{N}$ and $h_{N}$ denote the $N$ th partial sum of the series defining $g$ and $h$. Expanding $\left|g_{N}\right|^{p}$ as in the proof of Lemma 2.5 we get

$$
\left\|g_{N}\right\|_{p}^{p}=2^{-p} \sum_{\substack{0 \leq i_{1}, \ldots, i_{p} \leq N \\ \varepsilon_{1}, \ldots, \varepsilon_{p}= \pm 1}} b_{i_{1}} \cdots b_{i_{p}} \psi\left(\varepsilon_{1} i_{1}+\cdots+\varepsilon_{p} i_{p}\right),
$$

where $\psi(x)=1$ or 0 according as $x=0$ or $x \neq 0$. A similar expansion holds for $\left\|h_{N}\right\|_{p}^{p}$ and thus we get $\left\|g_{N}\right\|_{p} \leq\left\|h_{N}\right\|_{p}$. By $\|h\|_{p}<+\infty$ we have $\left\|h_{N}\right\|_{p} \rightarrow\|h\|_{p}$ as $N \rightarrow \infty$ (see [21, Chapter VII, Theorem 6.4]) and thus the partial sums $g_{N}, N \geq 1$ and consequently also the $(C, 1)$ means of the Fourier series of $g$ remain bounded in $L^{p}$ norm whence we get $\|g\|_{p}<+\infty$. (See the remark preceding Theorem 5.12 in [21, Chapter IV]). Thus $\left\|g_{N}\right\|_{p} \rightarrow\|g\|_{p}$ and our above claim follows.

Assume now that $f=\sum_{k=0}^{\infty} a_{k} \cos k x$ and $p$ satisfy the assumptions of the lemma. Set $\Delta_{N}=\sum_{2^{N}<k \leq 2^{N+1}} a_{k} \cos k x, N=0,1, \ldots$, and

$$
f_{1}=a_{0}+a_{1} \cos x+\sum_{N \text { even }} \Delta_{N}, \quad f_{2}=\sum_{N \text { odd }} \Delta_{N} .
$$

Since $f \in L^{2}$, the series defining $f_{1}$ and $f_{2}$ converge in $L^{2}$ norm and by $f \in L^{p}$ and the above remark we have $f_{1} \in L^{p}, f_{2} \in L^{p}$. Clearly, if $N$ is odd then $s_{k}\left(f_{1}\right)$ does not change for $2^{N} \leq k \leq 2^{N+1}$ and thus

$$
\begin{aligned}
2^{N} S_{2^{N}}\left(f_{1}\right) & =s_{2^{N}}\left(f_{1}\right)+s_{2^{N}+1}\left(f_{1}\right)+\cdots+s_{2^{N+1}-1}\left(f_{1}\right) \\
& =2^{N+1} \sigma_{2^{N+1}-1}\left(f_{1}\right)-2^{N} \sigma_{2^{N}-1}\left(f_{1}\right),
\end{aligned}
$$

whence

$$
\left|s_{2^{N}}\left(f_{1}\right)\right|=\left|s_{2^{N+1}}\left(f_{1}\right)\right| \leq 2\left|\sigma_{2^{N+1}-1}\left(f_{1}\right)\right|+\left|\sigma_{2^{N}-1}\left(f_{1}\right)\right| \leq 3 \sup _{k \geq 1}\left|\sigma_{k}\left(f_{1}\right)\right| .
$$

A similar remark holds if $f_{1}$ is replaced by $f_{2}$ and thus using (2.31), $f=$ $f_{1}+f_{2},\left\|f_{1}\right\|_{p} \leq\|f\|_{p},\left\|f_{2}\right\|_{p} \leq\|f\|_{p}$ and the Minkowski inequality, we get (2.30) with $A=6 K$.

\section{Proof of Theorem 1}

Assume that $\left(n_{k}\right)$ satisfies (1.9) with $\alpha>5 / 2$. Then we get, using the mean value theorem,

$$
\frac{n_{k+1}}{n_{k}} \geq \exp \left(\frac{1}{2 \sqrt{k+1}}(\log \log k)^{\alpha}\right) \geq 1+\frac{(\log \log k)^{\alpha}}{3 \sqrt{k}} \quad\left(k \geq k_{0}\right) .
$$

We now approximate the trigonometric functions $X_{k}=\cos n_{k} x$ by stepfunctions $Y_{k}$ as follows. Given $k \geq 1$, define $l=l(k)$ and $m=m(k)$ by $2^{l} \leq n_{k}<2^{l+1}, m=[l+5 \log k]$ where [ ] denotes integral part. Then set $Y_{k}=E\left(X_{k} \mid \mathscr{F}_{m(k)}\right)$ where $\mathscr{F}_{j}$ denotes the $\sigma$ field generated by the intervals $\left[2 \pi i 2^{-j}, 2 \pi(i+1) 2^{-j}\right)\left(0 \leq i \leq 2^{j}-1\right)$ and $E, P$ denote expectation and probability in the probability space $\left((0,2 \pi), \mathscr{B},(2 \pi)^{-1} \lambda\right)$. Clearly

$$
\left|X_{k}-Y_{k}\right| \leq n_{k} \cdot 2 \pi 2^{-m} \leq 4 \pi \cdot 2^{-5 \log k} \ll k^{-3},
$$


where $\ll$ means the same as the $O$ notation. Let us divide the set of positive integers into consecutive blocks $\Delta_{1}, \Delta_{1}^{\prime}, \Delta_{2}, \Delta_{2}^{\prime}, \ldots$ such that

$$
\left|\Delta_{k}\right|=k^{4}, \quad\left|\Delta_{k}^{\prime}\right|=k^{3},
$$

where $|A|$ denotes, for any set $A \subset \mathbf{R}$, the number of integers contained in $A$. Set

$$
\begin{gathered}
T_{k}=\sum_{\nu \in \Delta_{k}} X_{\nu}, \quad T_{k}^{\prime}=\sum_{\nu \in \Delta_{k}^{\prime}} X_{\nu}, \\
D_{k}=\sum_{\nu \in \Delta_{k}} Y_{\nu}, \quad D_{k}^{\prime}=\sum_{\nu \in \Delta_{k}^{\prime}} Y_{\nu}, \quad \bar{D}_{k}=D_{k}-E\left(D_{k} \mid \mathscr{G}_{k-1}\right),
\end{gathered}
$$

where $\mathscr{G}_{k-1}$ denotes the $\sigma$-field generated by $D_{1}, \ldots, D_{k-1}$.

Lemma 3.1. We have

$$
\left|E\left(D_{k} \mid \mathscr{G}_{k-1}\right)\right|=O\left(k^{-2}\right), \quad E\left(D_{k}^{2} \mid \mathscr{G}_{k-1}\right)=\frac{1}{2}\left|\Delta_{k}\right|+O\left(k^{-2}\right),
$$

where the constants implied by the $O$ are absolute.

Proof. Let $p=p(k)$ and $q=q(k)$ denote the largest integer of the block $\Delta_{k-1}$ and the smallest integer of the block $\Delta_{k}$, respectively. Let $l$ be the integer defined by $2^{l} \leq n_{p}<2^{l+1}$ and set $m=[l+5 \log p]$. Clearly, each $Y_{\nu}$, $1 \leq \nu \leq p$, is $\mathscr{F}_{m}$ measurable and thus $\mathscr{G}_{k-1} \subset \mathscr{F}_{m}$. Thus to prove Lemma 3.1 it suffices to show that for every atom $[a, b)$ of $\mathscr{F}_{m}$ we have

$$
\begin{aligned}
& (b-a)^{-1}\left|\int_{a}^{b} D_{k} d x\right|=O\left(k^{-2}\right) \\
& (b-a)^{-1} \int_{a}^{b} D_{k}^{2} d x=\frac{1}{2}\left|\Delta_{k}\right|+O\left(k^{-2}\right) .
\end{aligned}
$$

Now by (3.2), (3.3)

$$
\left|D_{k}-T_{k}\right| \ll \sum_{\nu \in \Delta_{k}} \nu^{-3} \leq \sum_{\nu=(k-1)^{4}}^{\infty} \nu^{-3} \ll k^{-8}
$$

and thus by $\left|T_{k}\right| \leq k^{4}$ we get

$$
\left|D_{k}^{2}-T_{k}^{2}\right| \ll k^{-4}
$$

Here, and in the rest of the proof of the lemma, the constants implied by $\ll$ are absolute. Clearly $q=\sum_{i \leq k-1}\left(i^{4}+i^{3}\right)+1 \leq 2 k^{5}, q-p=(k-1)^{3}$ and thus by (3.1)

$$
\begin{aligned}
\frac{n_{q}}{n_{p}} & \geq \prod_{j=p}^{q-1}\left(1+\frac{(\log \log j)^{\alpha}}{3 \sqrt{j}}\right) \geq \prod_{j=p}^{q-1}\left(1+\frac{1}{\sqrt{j}}\right) \geq\left(1+\frac{1}{\sqrt{q}}\right)^{q-p} \\
& \geq \exp \left(\frac{1}{2}(q-p) / \sqrt{q}\right) \geq \exp \left(\frac{1}{8} \sqrt{k}\right) \quad\left(k \geq k_{0}\right) .
\end{aligned}
$$

Hence using Lemma 2.6, relations (3.1), (3.3), (3.7), $b-a=2 \pi 2^{-m}$, and $2^{m} \leq 2^{l} p^{5} \leq n_{p} p^{5} \leq n_{p} k^{25}$, we get

$$
\left|(b-a)^{-1} \int_{a}^{b} T_{k} d x\right| \ll 2^{m} k^{4} / n_{q} \ll\left(n_{p} / n_{q}\right) k^{30} \ll k^{-2}
$$


and

$$
\left|(b-a)^{-1} \int_{a}^{b} T_{k}^{2} d x-\frac{1}{2}\right| \Delta_{k}|| \ll 2^{m} k^{12} k^{5} / n_{q} \ll\left(n_{p} / n_{q}\right) k^{42} \ll k^{-2} .
$$

Now (3.4) follows from (3.8), (3.9), (3.5), and (3.6).

Put $U_{k}=E\left(D_{k} \mid \mathscr{G}_{k-1}\right)$ then

$$
E\left(\bar{D}_{k}^{2} \mid \mathscr{G}_{k-1}\right)=E\left(\left(D_{k}-U_{k}\right)^{2} \mid \mathscr{G}_{k-1}\right)=E\left(D_{k}^{2} \mid \mathscr{G}_{k-1}\right)-U_{k}^{2},
$$

since $U_{k}$ is $\mathscr{G}_{k-1}$ measurable. Thus Lemma 3.1 and (3.5) imply

$$
E\left(\bar{D}_{k}^{2} \mid \mathscr{G}_{k-1}\right)=\frac{1}{2}\left|\Delta_{k}\right|+O\left(k^{-2}\right)
$$

and

$$
\left|\bar{D}_{k}-T_{k}\right|=O\left(k^{-2}\right),
$$

where the constants implied by the $O$ are absolute. Now we state our key lemma.

Lemma 3.2. Let

$$
s_{n}^{2}=\sum_{k=1}^{n} E\left(\bar{D}_{k}^{2} \mid \mathscr{G}_{k-1}\right) \text {. }
$$

Then for any nondecreasing function $\varphi:(0, \infty) \rightarrow(0, \infty)$ we have

$$
P\left(\sum_{k=1}^{n} \bar{D}_{k}>s_{n} \varphi\left(s_{n}^{2}\right) \text { i.o. }\right)=0 \text { or } 1
$$

depending on whether the integral in (2.20) converges or diverges. Moreover, for any nondecreasing function $\varphi:(0, \infty) \rightarrow(0, \infty)$ satisfying $(2.21)$ we have

$$
P\left(\sum_{k=1}^{n} \bar{D}_{k}>s_{n} \varphi\left(s_{n}^{2}\right) \text { i.o. }\right)=P\left(\sum_{k=1}^{n} \bar{D}_{k}+\tau_{n}>\hat{s}_{n} \varphi\left(\hat{s}_{n}^{2}\right) \text { i.o. }\right)
$$

for any sequences $\left\{\tau_{n}, n \geq 1\right\}$ and $\left\{\hat{s}_{n}, n \geq 1\right\}$ of r.v.'s such that

$$
\begin{gathered}
\tau_{n}=O\left(s_{n} /\left(\log \log s_{n}\right)^{1 / 2}\right) \quad \text { a.s., } \\
\hat{s}_{n}=s_{n}\left(1+O\left(\left(\log \log s_{n}\right)^{-1}\right)\right) \quad \text { a.s. }
\end{gathered}
$$

Remark. Relation (3.14) expresses the fact that the probability

$$
P\left(\sum_{k=1}^{n} \bar{D}_{k}>s_{n} \varphi\left(s_{n}^{2}\right) \text { i.o. }\right)
$$

does not change at small perturbations of $\sum_{k=1}^{n} \bar{D}_{k}$ and $s_{n}$. Actually, it suffices to prove the statement for $\tau_{n}=0$ since the convergence or divergence of the integral in (2.20) is not changed if $\varphi$ is replaced by $\varphi \pm C / \varphi$ for any constant $C>0$ and by (3.15), (3.16), and (2.21) we have

$$
P\left(\sum_{k=1}^{n} \bar{D}_{k}+\tau_{n}>\hat{s}_{n} \varphi\left(\hat{s}_{n}^{2}\right) \text { i.o. }\right)=P\left(\sum_{k=1}^{n} \bar{D}_{k}>\hat{s}_{n}\left(\varphi\left(\hat{s}_{n}^{2}\right)+O\left(\varphi\left(\hat{s}_{n}^{2}\right)^{-1}\right)\right) \text { i.o. }\right) \text {. }
$$


Proof of Lemma 3.2. Clearly, $\left\{\bar{D}_{k}, \mathscr{G}_{k}, k \geq 1\right\}$ is a martingale difference sequence with finite variances and thus by Corollary 2.8 it suffices to prove that

$$
\sum_{k \geq 1} \frac{\left(\log \log s_{k}\right)^{3}}{s_{k}^{2}} E\left(\bar{D}_{k}^{2} I\left(\left|\bar{D}_{k}\right| \geq s_{k}\left(\log \log s_{k}\right)^{-3 / 2}\right) \mid \mathscr{G}_{k-1}\right)<+\infty \quad \text { a.s. }
$$

To verify (3.17) we first observe that by (3.12), (3.10), and (3.3) we have

$$
s_{n}^{2}=\frac{1}{2} \sum_{k=1}^{n}\left|\Delta_{k}\right|+O(1)=\frac{1}{10} n^{5}+O\left(n^{4}\right) .
$$

Next we note that setting $\rho_{k}=s_{k}\left(\log \log s_{k}\right)^{-3 / 2}$ we have, for any integer $p_{k} \geq 2$,

$$
E\left(\bar{D}_{k}^{2} I\left(\left|\bar{D}_{k}\right| \geq \rho_{k}\right) \mid \mathscr{G}_{k-1}\right) \leq \rho_{k}^{-\left(p_{k}-2\right)} E\left(\left|\bar{D}_{k}\right|^{p_{k}} \mid \mathscr{G}_{k-1}\right)
$$

since $\rho_{k}$ is $\mathscr{G}_{k-1}$ measurable. Hence a sufficient condition for (3.17) is

$$
\sum_{k \geq 1} \frac{\left(\log \log s_{k}\right)^{3 p_{k} / 2}}{s_{k}^{p_{k}}} E\left(\left|\bar{D}_{k}\right|^{p_{k}} \mid \mathscr{G}_{k-1}\right)<+\infty \text { a.s., }
$$

which, in view of (3.18) and the Beppo Levi theorem, will be proved if we show that

$$
\sum_{k \geq 1} \frac{(8 \log \log k)^{3 p_{k} / 2}}{k^{5 p_{k} / 2}} E\left(\left|\bar{D}_{k}\right|^{p_{k}}\right)<+\infty .
$$

We now choose $p_{k}=2[\log \log k]$ and use Lemma 2.5 to get

$$
E\left(\left|T_{k}\right|^{p_{k}}\right) \leq 2\left(576 p_{k} \log p_{k}\right)^{p_{k}} k^{4} \frac{\left(k^{5 / 2}\right)^{\left(p_{k}-2\right)}}{c_{k}^{p_{k}-2}},
$$

where $c_{k}=(\log \log k)^{\alpha} / 4$. (To verify the assumptions of Lemma 2.5 in the present case note that by (3.1) we have $n_{j+1} / n_{j} \geq 1+c / \sqrt{j}$ for $j \in \Delta_{k}$ with $c=c_{k}$ and also

$$
c_{k}\left(k^{4} p_{k}\right)^{1 / 2} \leq\left(\sum_{i=1}^{k} i^{4}+\sum_{i=1}^{k-1} i^{3}\right)^{1 / 2} \leq c_{k} k^{4} / 48
$$

for $k \geq k_{0}$.) (3.11) and the Minkowski inequality show that (3.20) remains valid, with an extra factor $2^{p_{k}}$ on the right-hand side, if $T_{k}$ on the left-hand side is replaced by $\bar{D}_{k}$. Thus the $k$ th term of the series in (3.19) is

$$
\begin{aligned}
& \leq \frac{(8 \log \log k)^{3 p_{k} / 2}}{k^{5 p_{k} / 2}} 2^{2 p_{k}}\left(576 p_{k} \log p_{k}\right)^{p_{k}} k^{4} \frac{\left(k^{5 / 2}\right)^{\left(p_{k}-2\right)}}{c_{k}^{p_{k}-2}} \\
& \leq \frac{1}{k}(8 \log \log k)^{3 p_{k} / 2} \frac{\left(576 p_{k}^{1+\varepsilon}\right)^{p_{k}} 16^{p_{k}}}{(\log \log k)^{\alpha\left(p_{k}-2\right)}} \\
& \leq \frac{1}{k} \frac{\left(2^{37}\right)^{\log \log k}}{(\log \log k)^{(2 \alpha-5-2 \varepsilon) \log \log k-2 \alpha}} \leq \frac{1}{k} \frac{1}{(\log \log k)^{(2 \alpha-5-3 \varepsilon) \log \log k}} \\
& \leq \frac{1}{k(\log k)^{2}} \quad\left(k \geq k_{0}\right)
\end{aligned}
$$


for any sufficiently small $\varepsilon>0$ since $\alpha>5 / 2$. Thus (3.19) is verified and the proof of Lemma 3.2 is completed.

Observe now that by (3.3) the sequence $\left\{\sqrt{2} k^{-3 / 2} T_{k}^{\prime}\right\}$ is an orthonormal system and thus the Rademacher-Mensov convergence theorem (see e.g. [21, Chapter XIII, Theorem 10.21]) implies that $\sum_{k>1} k^{-2}(\log k)^{-2} T_{k}^{\prime}$ is a.s. convergent. Hence by the Kronecker lemma and (3.18) we get

$$
\sum_{k=1}^{n} T_{k}^{\prime}=O\left(n^{2} \log ^{2} n\right)=O\left(s_{n} /\left(\log \log s_{n}\right)^{1 / 2}\right) \quad \text { a.s. }
$$

The last relation, together with (3.11), yields

$$
\sum_{k=1}^{n}\left(T_{k}+T_{k}^{\prime}\right)=\sum_{k=1}^{n} \bar{D}_{k}+O\left(s_{n} /\left(\log \log s_{n}\right)^{1 / 2}\right) \text { a.s. }
$$

Moreover, setting $N_{k}=\sum_{i=1}^{k}\left(i^{4}+i^{3}\right),(3.3)$ and (3.18) yield

$$
\sqrt{N_{k} / 2}=s_{k}\left(1+O\left(\left(\log \log s_{k}\right)^{-1}\right)\right) \text { a.s. }
$$

From (3.22), (3.23), and Lemma 3.2 (cf. also the perturbational statement (3.14)) it follows that for any nondecreasing function $\varphi:(0, \infty) \rightarrow(0, \infty)$ satisfying (2.21) and any sequence $\left\{\tau_{k}, k \geq 1\right\}$ of r.v.'s satisfying (3.15) we have

$$
P\left(\sum_{j=1}^{k}\left(T_{j}+T_{j}^{\prime}\right)+\tau_{k}>\sqrt{N_{k} / 2} \varphi\left(N_{k} / 2\right) \text { i.o. }\right)=0 \text { or } 1
$$

depending on whether $I(\varphi)$ converges or diverges. Here, for brevity, we introduced the symbol $I(\varphi)$ for the integral in $(2.20)$. Since $I(\varphi)<+\infty$ iff $I\left(\varphi_{1}\right)<+\infty$, where $\varphi_{1}(t)=\varphi(t / 2)$, we have proved the following lemma.

Lemma 3.3. Let $S_{N}=\sum_{j=1}^{N} \cos n_{j} x$. Then for any nondecreasing function $\varphi:(0, \infty) \rightarrow(0, \infty)$ satisfying $(2.21)$ we have

$$
P\left(S_{N_{k}}>\sqrt{N_{k} / 2} \varphi\left(N_{k}\right) \text { i.o. }\right)=0 \text { or } 1
$$

depending on whether $I(\varphi)$ converges or diverges. Moreover, the probability in (3.24) does not change if we replace $S_{N_{k}}$ by $S_{N_{k}}+\tau_{k}$, where $\left\{\tau_{k}, k \geq 1\right\}$ is any sequence of r.v.'s satisfying $\tau_{k}=O\left(\left(N_{k} / \log \log N_{k}\right)^{1 / 2}\right)$ a.s.

To complete the proof of Theorem 1 it remains to show that for any nondecreasing function $\varphi:(0, \infty) \rightarrow(0, \infty)$ satisfying (2.21) we have

$$
P\left(S_{N}>\sqrt{N / 2} \varphi(N) \text { i.o. }\right)=P\left(S_{N_{k}}>\sqrt{N_{k} / 2} \varphi\left(N_{k}\right) \text { i.o. }\right) .
$$

This statement will be an easy consequence of the following lemma.

Lemma 3.4. Let $M_{k}=\max _{N_{k} \leq j \leq N_{k+1}}\left|S_{j}-S_{N_{k}}\right|$. Then

$$
M_{k}=O\left(\left(N_{k} / \log \log N_{k}\right)^{1 / 2}\right) \text { a.s. }
$$

To deduce (3.25) from Lemma 3.4 note that if $I(\varphi)=+\infty$ then the righthand side of (3.25) and thus also the left-hand side is 1 and hence we may assume $I(\varphi)<+\infty$. Then the right-hand side of (3.25) is 0 and by (3.26) and the last statement of Lemma 3.3 we have $S_{N_{k}}+M_{k} \leq \sqrt{N_{k} / 2} \varphi\left(N_{k}\right)$ a.s. for 
$k>k_{0}$. But then for any $N_{k} \leq N \leq N_{k+1}, k \geq k_{0}$ we have $S_{N} \leq S_{N_{k}}+M_{k} \leq$ $\sqrt{N_{k} / 2} \varphi\left(N_{k}\right) \leq \sqrt{N / 2} \varphi(N)$ i.e., the left-hand side of (3.23) is also 0 .

Proof of Lemma 3.4. Set

$$
Z_{k}=\sum_{j=N_{k}+1}^{N_{k+1}} \cos n_{j} x, \quad p(k)=\max \left\{i: n_{i} \leq 2^{k}\right\}, \quad H=\{p(1), p(2), \ldots\} .
$$

Clearly

$$
M_{k} \leq \max _{N_{k} \leq j \leq N_{k+1}}\left|S_{j}-S_{N_{k}}\right|+\max _{\left\{i: p(i) \leq N_{k+1}\right\}}|p(i+1)-p(i)|:=J_{1}+J_{2} .
$$

By (3.1) we have for $k \geq k_{0}$

$$
\begin{aligned}
2 & \geq n_{p(k+1)} / n_{p(k)+1} \geq \prod_{m=p(k)+1}^{p(k+1)-1}\left(1+\frac{(\log \log m)^{\alpha}}{3 \sqrt{m}}\right) \\
& \geq 1+\sum_{m=p(k)+1}^{p(k+1)-1} \frac{(\log \log m)^{\alpha}}{3 \sqrt{m}} \geq 1+(p(k+1)-p(k)-1) \frac{(\log \log p(k+1))^{\alpha}}{3 \sqrt{p(k+1)}},
\end{aligned}
$$

whence $p(k+1) / p(k) \rightarrow 1$ and

$$
p(k+1)-p(k) \ll p(k)^{1 / 2}(\log \log p(k))^{-\alpha} .
$$

Thus for $J_{2}$ in (3.27) we get, using $N_{k+1} / N_{k} \rightarrow 1$ and $\alpha \geq 1 / 2$,

$$
\left|J_{2}\right| \ll\left(N_{k} / \log \log N_{k}\right)^{1 / 2} \text { a.s. }
$$

On the other hand, applying Lemma 2.10 for $f=Z_{k}$ we get for any even integer $p \geq 2$

$$
E\left|J_{1}\right|^{p} \leq A^{p} E\left|Z_{k}\right|^{p},
$$

where $A>1$ is an absolute constant. Hence choosing $p=p_{k}=2[\log \log k]$ and using (3.29), Lemma 2.5, $N_{k} \sim k^{5} / 5, N_{k+1}-N_{k} \sim k^{4}$, and the Markov inequality, we get, setting $c_{k}=(\log \log k)^{\alpha} / 4$,

$$
\begin{aligned}
P\left(\left|J_{1}\right|\right. & \left.\geq \sqrt{N_{k} / \log \log N_{k}}\right) \leq \frac{\left(\log \log N_{k}\right)^{p_{k} / 2}}{N_{k}^{p_{k} / 2}} A^{p_{k}} E\left|Z_{k}\right|^{p_{k}} \\
\leq & \frac{(12 \log \log k)^{p_{k} / 2}}{k^{5 p_{k} / 2}} 4 A^{p_{k}}\left(576 p_{k} \log p_{k}\right)^{p_{k}} k^{4} \frac{\left(k^{5 / 2}\right)^{\left(p_{k}-2\right)}}{c_{k}^{p_{k}-2}} .
\end{aligned}
$$

Observe that for $k \geq k_{0}$ the last expression in (3.30) is smaller than the first expression in (3.21) and thus it cannot exceed $k^{-1}(\log k)^{-2}$. Hence (3.30) and the Borel-Cantelli lemma imply

$$
\left|J_{1}\right| \ll\left(N_{k} / \log \log N_{k}\right)^{1 / 2} \text { a.s. }
$$

Now Lemma 3.4 follows from (3.27), (3.30), and (3.31).

The just completed proof of Theorem 1 yields, with trivial modifications, various related limit theorems for $\left(\cos n_{k} x\right)$ under condition (1.9). For example, replacing Lemma 2.7 in the proof of Theorem 1 with the martingale version of the Chung test formulated in Remark 2.9 we get that $\left(\cos n_{k} x\right)$ satisfies the 
Chung test (1.24) $-(1.25)$ if (1.9) holds with $\alpha>7 / 2$. Similarly, using the a.s. invariance principle mentioned in Remark 2.9 we get, without any difficulty, the a.s. invariance principle $(1.26)$ for $\left(\cos n_{k} x\right)$.

\section{Proof of Theorem 2}

Lemma 4.1. We have

$$
\begin{aligned}
& \frac{1}{2 \pi} \int_{0}^{2 \pi}\left(\sum_{j=1}^{n} \cos j x\right)^{3} d x=\frac{3}{8} N^{2}+O(N), \\
& \frac{1}{2 \pi} \int_{0}^{2 \pi}\left(\sum_{j=1}^{n} \cos j x\right)^{4} d x=\frac{1}{3} N^{3}+O\left(N^{2}\right) .
\end{aligned}
$$

Proof. We prove (4.2). As in the proof of Lemma 2.4, the left side of (4.2) equals $1 / 16$ times the number of solutions of

$$
\pm j_{1} \pm j_{2} \pm j_{3} \pm j_{4}=0, \quad 1 \leq j_{1}, j_{2}, j_{3}, j_{4} \leq N .
$$

Simple calculations show that the equation

$$
\pm j_{1} \pm j_{2}=\nu, \quad 1 \leq j_{1}, j_{2} \leq N
$$

has $2 N, 2 N-1-|\nu|, 2 N+1-|\nu|$, or 0 solutions according as $\nu=0$, $1 \leq|\nu| \leq N, N+1 \leq|\nu| \leq 2 N$, or $|\nu|>2 N$. Thus the number of solutions of (4.3) equals

$$
(2 N)^{2}+\sum_{1 \leq|\nu| \leq N}(2 N-1-|\nu|)^{2}+\sum_{N+1 \leq|\nu| \leq 2 N}(2 N+1-|\nu|)^{2}=\frac{16 N^{3}}{3}+O\left(N^{2}\right)
$$

and $(4.2)$ is proved.

Let $a_{k}=2^{k^{2}}, m_{k}=\left[A k /(\log \log k)^{2 \alpha}\right](k \geq 3) ; m_{1}=m_{2}=0$ and $M_{k}=$ $\sum_{i=1}^{k} m_{i}$ where $\alpha \geq 1 / 2$ and $A$ is an absolute constant with $0<A \leq 10^{-6}$. Using the easily verifiable formula

$$
\sum_{k=3}^{n} \frac{k^{\beta}}{(\log \log k)^{\gamma}}=\frac{1}{\beta+1} \frac{n^{\beta+1}}{(\log \log n)^{\gamma}}\left(1+O\left((\log \log n)^{-1}\right)\right) \quad(\beta \neq-1)
$$

we get

$$
M_{k}=\frac{A}{2} \frac{k^{2}}{(\log \log k)^{2 \alpha}}\left(1+O\left((\log \log k)^{-1}\right)\right) .
$$

Let $I_{k}=\left\{a_{k}, 2 a_{k}, \ldots, m_{k} a_{k}\right\}$; clearly the sets $I_{k}, k=1,2, \ldots$, are disjoint. Define the sequence $\left(n_{k}\right)$ by $\left(n_{k}\right)=\bigcup_{j=1}^{\infty} I_{j}$. We prove that $\left(\cos n_{k} x\right)$ satisfies the requirements of Theorem 2 . As a first step we show that

$$
n_{k} \succ \exp \left\{\frac{1}{4 \sqrt{A}} \sqrt{k}(\log \log k)^{\alpha}\right\} \text {. }
$$


Indeed, if $M_{k-1}<j<M_{k}$ then setting $i=j-M_{k-1}$ and using (4.5), $M_{k} / M_{k-1} \rightarrow 1$ and the mean value theorem we get

$$
\begin{aligned}
\frac{n_{j+1}}{n_{j}} & =1+\frac{1}{i} \geq 1+\frac{1}{m_{k}} \geq 1+\frac{(\log \log k)^{2 \alpha}}{A k} \\
& \geq 1+\frac{\left(\log \log M_{k}\right)^{\alpha}}{2 \sqrt{A} \sqrt{M_{k-1}}} \geq 1+\frac{(\log \log j)^{\alpha}}{2 \sqrt{A} \sqrt{j}} \geq \exp \left(\frac{(\log \log j)^{\alpha}}{4 \sqrt{A} \sqrt{j}}\right) \\
& \geq \exp \left(\frac{1}{4 \sqrt{A}}\left\{\sqrt{j+1}(\log \log (j+1))^{\alpha}-\sqrt{j}(\log \log j)^{\alpha}\right\}\right)
\end{aligned}
$$

for $j \geq j_{0}$. On the other hand, if $j=M_{k}$ then $n_{j+1} / n_{j}=a_{k+1} /\left(m_{k} a_{k}\right) \geq 2 \geq$ $1+1 / m_{k}$ i.e., we get the same lower bound for $n_{j+1} / n_{j}$ as above. Thus we proved (4.6). Set now $q_{k}=a_{k+1} / a_{k}$ and

$$
X_{k}=\sum_{j=M_{k-1}+1}^{M_{k}} \cos n_{j} x, \quad f_{k}(x)=\sum_{j=1}^{m_{k}} \cos j x .
$$

Further let $\rho_{k}(x) \quad(0 \leq x \leq 2 \pi)$ be the function which equals $2 \pi j / q_{k}$ provided $2 \pi j / q_{k} \leq\left(a_{k} x\right)_{2 \pi}<2 \pi(j+1) / q_{k}$ for some integer $0 \leq j \leq q_{k}-1$; here $(t)_{2 \pi}$ denotes the residue of $t \bmod 2 \pi$. Clearly, $\rho_{k}(x)$ is constant on each interval $\left[2 \pi j / a_{k+1}, 2 \pi(j+1) / a_{k+1}\right) \quad\left(0 \leq j \leq a_{k+1}-1\right)$ and is periodic with period $2 \pi / a_{k}$. Thus the functions $\rho_{k}(x), k=1,2, \ldots$, are independent r.v.'s over the probability space $\left((0,2 \pi), \mathscr{B},(2 \pi)^{-1} \lambda\right)$. Further,

$$
\left|\rho_{k}(x)-\left(a_{k} x\right)_{2 \pi}\right| \leq 2 \pi / q_{k} \quad(0 \leq x \leq 2 \pi) .
$$

Now let $Y_{k}=f_{k}\left(\rho_{k}(x)\right)$, then using $X_{k}=f_{k}\left(a_{k} x\right)=f_{k}\left(\left(a_{k} x\right)_{2 \pi}\right),\left|f_{k}^{\prime}\right| \leq m_{k}^{2}$, (4.7), and the mean value theorem we get $\left|X_{k}-Y_{k}\right| \leq 2 \pi m_{k}^{2} / q_{k} \ll 2^{-k}$ which, in view of $E X_{k}=0$, implies

$$
\left|X_{k}-Z_{k}\right| \ll 2^{-k},
$$

where $Z_{k}=Y_{k}-E Y_{k}$. Moreover, the $Z_{k}$ are independent r.v.'s over the probability space $\left((0,2 \pi), \mathscr{B},(2 \pi)^{-1} \lambda\right)$. Since $\left|X_{k}\right| \leq m_{k}$, the last relation and the mean value theorem imply

$$
\left|X_{k}^{2}-Z_{k}^{2}\right| \ll 2^{-k} m_{k} \ll 2^{-k / 2}
$$

and similarly

$$
\left|X_{k}^{3}-Z_{k}^{3}\right| \ll 2^{-k / 2}, \quad\left|X_{k}^{4}-Z_{k}^{4}\right| \ll 2^{-k / 2} .
$$

Now using (4.5) and (4.9) we get

$$
\begin{aligned}
\sigma_{k}^{2}:=E Z_{k}^{2} & =E X_{k}^{2}+O\left(2^{-k / 2}\right)=\frac{1}{2} m_{k}+O\left(2^{-k / 2}\right), \\
s_{n}^{2} & :=\sum_{k=1}^{n} E Z_{k}^{2}=\frac{1}{2} M_{n}+O(1),
\end{aligned}
$$

whence we get by $(4.5)$

$$
s_{n}=\frac{\sqrt{A}}{2} \frac{n}{(\log \log n)^{\alpha}}\left(1+O\left((\log \log n)^{-1}\right)\right) .
$$


Further using Lemma 4.1, $X_{k}=f_{k}\left(a_{k} x\right)$, the periodicity of $f_{k},(4.10)$, and (4.4) we get

$$
\begin{aligned}
\sum_{k=1}^{n} E Z_{k}^{3} & =\sum_{k=1}^{n} E X_{k}^{3}+O(1)=\sum_{k=1}^{n} \frac{1}{2 \pi} \int_{0}^{2 \pi} f_{k}^{3}\left(a_{k} x\right) d x+O(1) \\
& =\sum_{k=1}^{n} \frac{1}{2 \pi} \int_{0}^{2 \pi} f_{k}^{3}(x) d x+O(1)=\sum_{k=1}^{n} \frac{3}{8} m_{k}^{2}+O\left(\sum_{k=1}^{n} m_{k}\right) \\
& =\frac{A^{2}}{8} \frac{n^{3}}{(\log \log n)^{4 \alpha}}\left(1+O\left((\log \log n)^{-1}\right)\right) .
\end{aligned}
$$

Similarly

$$
\begin{aligned}
& \sum_{k=1}^{n} E Z_{k}^{4}=\frac{A^{3}}{12} \frac{n^{4}}{(\log \log n)^{6 \alpha}}\left(1+O\left((\log \log n)^{-1}\right)\right) \\
& \sum_{k=1}^{n}\left(E Z_{k}^{2}\right)^{2}=\frac{A^{2}}{12} \frac{n^{3}}{(\log \log n)^{4 \alpha}}\left(1+O\left((\log \log n)^{-1}\right)\right)
\end{aligned}
$$

Set

$$
\lambda_{n}=4 \sqrt{A}(\log \log n)^{-\alpha} .
$$

Then by (4.8), $\left|X_{k}\right| \leq m_{k}$, and (4.13) we have

$$
\left|Z_{n}\right| \leq \lambda_{n} s_{n} \quad\left(n \geq n_{0}\right) \text {. }
$$

Also by (4.13), $\alpha \geq 1 / 2$, and $A \leq 10^{-6}$ we have

$$
\lambda_{n} \leq \frac{1}{200}\left(\log \log s_{n}\right)^{-1 / 2} \quad\left(n \geq n_{0}\right) .
$$

Hence applying Feller's general upper-lower class criterion (see [8, Theorems 1 and 11]) for the independent sequence $\left\{Z_{n}, n \geq 1\right\}$ we get that for any positive nondecreasing sequence $\psi_{n}$ we have

$$
P\left(\sum_{k=1}^{n} Z_{k}>s_{n} \psi_{n} \text { i.o. }\right)=0 \text { or } 1
$$

depending on whether

$$
\sum_{n \geq 1} \frac{\sigma_{n}^{2}}{s_{n}^{2}} \psi_{n} \exp \left\{-\frac{1}{2} \psi_{n}^{2}\left(1+Q_{n}\left(\psi_{n}\right)\right)\right\}<+\infty \text { or }=+\infty .
$$

Here $Q_{n}(x)=\sum_{\nu=1}^{\infty} q_{n, \nu} x^{\nu}$ is a function analytic for $|x|<1 /\left(12 \lambda_{n}\right)$ whose coefficients can be explicitly calculated from the moments of the $Z_{i}$ 's. Actually $q_{n, \nu}$ depends on the first $\nu+2$ moments of $Z_{1}, \ldots, Z_{n}$, e.g.

$$
\begin{aligned}
& q_{n, 1}=\frac{1}{3 s_{n}^{3}} \sum_{k=1}^{n} E Z_{k}^{3}, \\
& q_{n, 2}=\frac{1}{12 s_{n}^{4}} \sum_{k=1}^{n} E Z_{k}^{4}-\frac{1}{4 s_{n}^{4}} \sum_{k=1}^{n}\left(E Z_{k}^{2}\right)^{2}-\frac{1}{4 s_{n}^{6}}\left(\sum_{k=1}^{n} E Z_{k}^{3}\right)^{2} .
\end{aligned}
$$


Further

$$
\left|q_{n, \nu}\right| \leq \frac{1}{7}\left(12 \lambda_{n}\right)^{\nu} \quad(n \geq 1, \nu \geq 1) .
$$

Using (4.13)-(4.16), (4.20), and (4.21) we get

$$
\begin{aligned}
& q_{n, 1}=\frac{\sqrt{A}}{3}(\log \log n)^{-\alpha}\left(1+O\left((\log \log n)^{-1}\right)\right), \\
& q_{n, 2}=-\frac{5 A}{36}(\log \log n)^{-2 \alpha}\left(1+O\left((\log \log n)^{-1}\right)\right),
\end{aligned}
$$

and

$$
\left|q_{n, \nu}\right| \leq \frac{(48 \sqrt{A})^{\nu}}{7}(\log \log n)^{-\nu \alpha} \quad(n \geq 1, \nu \geq 1) .
$$

From (4.21) we easily get

$$
\left|Q_{n}(x)\right| \leq 1 / 4, \quad\left|Q_{n}^{\prime}(x)\right| \leq 12 \lambda_{n} \quad \text { for }\left|\lambda_{n} x\right| \leq 1 / 24 .
$$

We also note the fact that if $4^{-1}(\log \log n)^{1 / 2} \leq \psi_{n} \leq 4(\log \log n)^{1 / 2}$ (actually, it suffices to assume $\left.\lambda_{n} \psi_{n} \leq 1 / 48\right)$ then replacing $\psi_{n}$ by $\psi_{n} \pm C / \psi_{n} \quad(C>0$ is an arbitrary constant), the convergence or divergence of the series (4.19) is not affected. (This is observed in [8] and easily verified since (4.25) and the mean value theorem show that replacing $\psi_{n}$ by $\psi_{n} \pm C / \psi_{n}$ the exponent in (4.19) changes by $O(1)$ if $\lambda_{n} \psi_{n} \leq 1 / 48$.) This remark implies that the probability in (4.18) does not change if $\sum_{k=1}^{n} Z_{k}$ is replaced by $\sum_{k=1}^{n} Z_{k}+\tau_{n}$ where $\left\{\tau_{n}, n \geq 1\right\}$ is any sequence of r.v.'s such that $\tau_{n}=O\left(s_{n} /\left(\log \log s_{n}\right)^{1 / 2}\right)$. Now letting $S_{N}=\sum_{j \leq N} \cos n_{j} x$, we have by (4.8) and (4.13)

$$
S_{M_{k}}=\sum_{i=1}^{k} X_{i}=\sum_{i=1}^{k} Z_{i}+O(1)=\sum_{i=1}^{k} Z_{i}+O\left(s_{k} /\left(\log \log s_{k}\right)^{1 / 2}\right)
$$

and thus we proved the following result.

Lemma 4.2. Assume $\varphi_{n}$ is a nondecreasing sequence satisfying

$$
2^{-1}(\log \log n)^{1 / 2} \leq \varphi_{n} \leq 2(\log \log n)^{1 / 2}
$$

Then

$$
P\left(S_{M_{k}}>s_{k} \varphi_{M_{k}} \text { i.o. }\right)=0 \text { or } 1
$$

depending on whether

$$
\sum_{k \geq 1} \frac{\sigma_{k}^{2}}{s_{k}^{2}} \varphi_{M_{k}} \exp \left\{-\frac{1}{2} \varphi_{M_{k}}^{2}\left(1+Q_{k}\left(\varphi_{M_{k}}\right)\right)\right\}<+\infty \quad \text { or }=+\infty .
$$

Here

$$
Q_{k}(x)=\sum_{\nu=1}^{\infty} \frac{c_{k, \nu}}{(\log \log k)^{\nu \alpha}} x^{\nu}
$$

is a power series converging for $|x| \leq 10(\log \log n)^{\alpha}$ whose coefficients $c_{k, \nu}$ are explicitly calculable numbers with

(4.30) $c_{k, 1}=\frac{\sqrt{A}}{3}\left(1+O\left((\log \log k)^{-1}\right)\right), \quad c_{k, 2}=\frac{-5 A}{36}\left(1+O\left((\log \log k)^{-1}\right)\right)$, 
and

$$
\left|c_{k, \nu}\right| \leq \frac{(48 \sqrt{A})^{\nu}}{7} \quad(k \geq 1, \nu \geq 1) .
$$

In what follows we shall prove that

$$
P\left(S_{N}>\sqrt{N / 2} \varphi_{N} \text { i.o. }\right)=P\left(S_{M_{k}}>s_{k} \varphi_{M_{k}} \text { i.o. }\right)
$$

and we shall also write the sum (4.28) in a simpler form, not containing quantities depending explicitly on the sequence $M_{k}$. We break the argument into steps.

Lemma 4.3. Let the nondecreasing function $\varphi_{n}$ satisfy (4.26) and assume that $\varphi_{M_{k}}^{2}-\varphi_{M_{k-1}}^{2} \leq 1$. Then

$$
\left|\varphi_{i}^{2}\left(1+Q_{k}\left(\varphi_{i}\right)\right)-\varphi_{j}^{2}\left(1+Q_{k}\left(\varphi_{j}\right)\right)\right| \leq 2 \text { for } M_{k-1} \leq i \leq j \leq M_{k} .
$$

Proof. Clearly the left side of (4.32) is bounded by

$$
\left(\varphi_{j}^{2}-\varphi_{i}^{2}\right)\left(1+\left|Q_{k}\left(\varphi_{i}\right)\right|\right)+\varphi_{j}^{2}\left|Q_{k}\left(\varphi_{i}\right)-Q_{k}\left(\varphi_{j}\right)\right|
$$

By $\alpha \geq 1 / 2, A \leq 10^{-6},(4.5),(4.17)$, and the second inequality of (4.26) we have $\lambda_{k} \varphi_{i} \leq 1 / 24, \lambda_{k} \varphi_{j} \leq 1 / 24$, and thus (4.25) implies $\left|Q_{k}\left(\varphi_{i}\right)\right| \leq 1 / 4$. Hence the first term in (4.33) is $\leq\left(\varphi_{M_{k}}^{2}-\varphi_{M_{k-1}}^{2}\right) \cdot 3 / 2 \leq 3 / 2$. On the other hand, using (4.25) and the mean value theorem it follows that the second term in $(4.33)$ is

$$
\leq \varphi_{j}^{2} \cdot 12 \lambda_{k}\left(\varphi_{j}-\varphi_{i}\right) \leq \varphi_{j} \cdot 12 \lambda_{k}\left(\varphi_{j}^{2}-\varphi_{i}^{2}\right) \leq 12 \lambda_{k} \varphi_{j} \leq 1 / 2 .
$$

Hence (4.32) is proved.

Lemma 4.4. Assume that $\varphi_{n}$ is nondecreasing and satisfies (4.26). Then the series in (4.28) is equiconvergent with

$$
\sum_{n \geq 1} \frac{\varphi_{n}}{n} \exp \left\{-\frac{1}{2} \varphi_{n}^{2}\left(1+\widehat{Q}_{n}\left(\varphi_{n}\right)\right)\right\},
$$

where $\widehat{Q}_{n}=Q_{k}$ for $M_{k-1}<n \leq M_{k}$.

Proof. Let $\mathscr{H}$ denote the set of those integers $k \geq 1$ such that $\varphi_{M_{k}}^{2}-\varphi_{M_{k-1}}^{2} \leq 1$. Using the monotonicity of $\varphi_{n},(4.11),(4.12)$, Lemma $4.3, M_{k} / M_{k-1} \rightarrow 1$, and the fact that by (4.26) and (4.5) $\varphi_{M_{k}} / \varphi_{M_{k-1}}$ is bounded, we get for $k \in \mathscr{H}$,

$$
\begin{aligned}
& \sum_{M_{k-1}<n \leq M_{k}} \frac{\varphi_{n}}{n} \exp \left\{-\frac{1}{2} \varphi_{n}^{2}\left(1+\widehat{Q}_{n}\left(\varphi_{n}\right)\right)\right\} \\
& \asymp \sum_{M_{k-1}<n \leq M_{k}} \frac{\varphi_{M_{k}}}{M_{k}} \exp \left\{-\frac{1}{2} \varphi_{M_{k}}^{2}\left(1+Q_{k}\left(\varphi_{M_{k}}\right)\right)+O(1)\right\} \\
& \asymp \frac{M_{k}-M_{k-1}}{M_{k}} \varphi_{M_{k}} \exp \left\{-\frac{1}{2} \varphi_{M_{k}}^{2}\left(1+Q_{k}\left(\varphi_{M_{k}}\right)\right)\right\} \\
& \asymp \frac{\sigma_{k}^{2}}{s_{k}^{2}} \varphi_{M_{k}} \exp \left\{-\frac{1}{2} \varphi_{M_{k}}^{2}\left(1+Q_{k}\left(\varphi_{M_{k}}\right)\right)\right\},
\end{aligned}
$$

where $c_{k} \asymp d_{k}$ means that $c_{k} / d_{k}$ lies between positive constants independent of $k$. On the other hand, in the proof of Lemma 4.3 we saw that $\left|Q_{k}\left(\varphi_{n}\right)\right| \leq 1 / 2$ 
for any $M_{k-1} \leq n \leq M_{k}, k \geq 1$ (regardless whether $k \in \mathscr{H}$ ) and thus for any $k \notin \mathscr{H}$ the first sum in (4.35) is bounded by

$$
\sum_{M_{k-1}<n \leq M_{k}} \frac{\varphi_{M_{k}}}{M_{k-1}} \exp \left\{-\frac{1}{4} \varphi_{M_{k-1}}^{2}\right\} \ll \varphi_{M_{k}} \exp \left\{-C \varphi_{M_{k}}^{2}\right\} \ll \exp \left\{-\frac{C}{2} \varphi_{M_{k}}^{2}\right\}
$$

for some constant $C>0$; here again we used the boundedness of $\varphi_{M_{k}} / \varphi_{M_{k-1}}$. Since $\varphi_{M_{k}}^{2}-\varphi_{M_{k-1}}^{2}>1$ for $k \notin \mathscr{H}$, the terms of the sum

$$
\sum_{k \notin \mathscr{H}} \exp \left\{-\frac{C}{2} \varphi_{M_{k}}^{2}\right\}
$$

decrease at least exponentially and thus the sum of converges. Since $\sigma_{k}^{2} / s_{k}^{2} \leq 1$, the same argument shows that adding the terms of (4.28) for $k \notin \mathscr{H}$ we get a convergent series. From these facts, the equiconvergence of (4.28) and (4.34) follows immediately.

By (4.29) and the definition of $\widehat{Q}_{n}$ we have

$$
\widehat{Q}_{n}(x)=\sum_{\nu=1}^{\infty} \frac{c_{\bar{n}, \nu}}{(\log \log \bar{n})^{\alpha \nu}} x^{\nu},
$$

where $\bar{n}$ is defined by $M_{\bar{n}-1}<n \leq M_{\bar{n}}$. The sum (4.36) is similar to (4.29) but the coefficients on the right-hand side contain the powers of $(\log \log \bar{n})^{\alpha}$ instead of $(\log \log n)^{\alpha}$. As the following lemma shows, replacing $\log \log \bar{n}$ by $\log \log n$ in (4.36) will not affect the convergence or divergence of (4.34).

Lemma 4.5. Let $\varphi_{n}$ be a nondecreasing sequence satisfying (4.26). Then the series (4.34) is equiconvergent with

$$
\sum_{n \geq 1} \frac{\varphi_{n}}{n} \exp \left\{-\frac{1}{2} \varphi_{n}^{2}\left(1+Q_{n}^{*}\left(\varphi_{n}\right)\right)\right\}
$$

where

$$
Q_{n}^{*}(x)=\sum_{\nu=1}^{\infty} \frac{c_{\bar{n}, \nu}}{(\log \log n)^{\alpha \nu}} x^{\nu} .
$$

Proof. By (4.5) we have $\sqrt{n} \leq \bar{n} \leq n$ and thus $\log \log n-\log \log \bar{n} \leq 1$ for large enough $n$. Hence by the mean value theorem we get

$$
\left|(\log \log n)^{-\alpha \nu}-(\log \log \bar{n})^{-\alpha \nu}\right| \leq \alpha \nu 2^{\alpha \nu+1}(\log \log n)^{-\alpha \nu-1} \quad\left(n \geq n_{0}\right) .
$$

Thus using (4.31), (4.26), $\alpha \geq 1 / 2$, and $A \leq 10^{-6}$ we get

$$
\begin{aligned}
\varphi_{n}^{2}\left|\widehat{Q}_{n}\left(\varphi_{n}\right)-Q_{n}^{*}\left(\varphi_{n}\right)\right| & \leq \varphi_{n}^{2} \sum_{\nu=1}^{\infty}\left|c_{\bar{n}, \nu}\right| 2 \alpha \nu 2^{\alpha \nu}(\log \log n)^{-\alpha \nu-1} \varphi_{n}^{\nu} \\
& \leq \frac{2 \alpha}{7} \frac{\varphi_{n}^{2}}{\log \log n} \sum_{\nu=1}^{\infty} \nu\left(\frac{2^{\alpha} \cdot 48 \sqrt{A} \varphi_{n}}{(\log \log n)^{\alpha}}\right)^{\nu} \\
& \ll O(1) \sum_{\nu=1}^{\infty} \nu 2^{-\nu}=O(1)
\end{aligned}
$$

and Lemma 4.5 follows. 
The coefficients $c_{\bar{n}, \nu}$ in (4.38) depend on $n, \nu$ in a rather complicated way. However, as we shall not explicitly compute them (with the exception of the first two), it is worth changing the notation and to write

$$
Q_{n}^{*}(x)=\sum_{\nu=1}^{\infty} \frac{a_{n, \nu}}{(\log \log n)^{\alpha \nu}} x^{\nu},
$$

where, in view of (4.30), (4.31) and $\log \log \bar{n} \sim \log \log n$ we have

$$
\begin{aligned}
a_{n, 1} & =\frac{\sqrt{A}}{3}\left(1+O\left((\log \log n)^{-1}\right)\right), \\
a_{n, 2} & =-\frac{5 A}{36}\left(1+O\left((\log \log n)^{-1}\right)\right), \\
\left|a_{n, \nu}\right| & \leq \frac{(48 \sqrt{A})^{\nu}}{7} \quad(n \geq 1, \nu \geq 1) .
\end{aligned}
$$

Moreover, the convergence or divergence of the series (4.37) is not affected if we change the coefficients $a_{n, 1}$ and $a_{n, 2}$ in (4.39) by deleting the error terms $O\left((\log \log n)^{-1}\right)$ in (4.40). (Indeed, the error made by these deletions in $\varphi_{n}^{2} Q_{n}^{*}\left(\varphi_{n}\right)$ is $\ll \varphi_{n}^{3}(\log \log n)^{-\alpha-1}+\varphi_{n}^{4}(\log \log n)^{-2 \alpha-1}$ which is $O(1)$ by (4.26) and $\alpha \geq 1 / 2$.) Thus instead of (4.40) we can write

$$
a_{n, 1}=\frac{\sqrt{A}}{3}, \quad a_{n, 2}=-\frac{5 A}{36} .
$$

Lemma 4.6. Let $\varphi_{n}$ be a nondecreasing sequence satisfying (4.26). Then

$$
P\left(S_{N}>\sqrt{N / 2} \varphi_{N} \text { i.o. }\right)=P\left(S_{M_{k}}>s_{k} \varphi_{M_{k}} \text { i.o. }\right) .
$$

Proof. By !emma 4.2 the right-hand side of (4.43) is 0 or 1 according as the series (4.28) converges or diverges. Moreover, the remark following (4.25) shows that the right-hand side of (4.43) does not change if we replace $\varphi_{M_{k}}$ by $\varphi_{M_{k}} \pm$ $10 / \varphi_{M_{k}}$. Further, by (4.12) we have $s_{n}=\sqrt{M_{n} / 2}+O\left(M_{n}^{-1 / 2}\right)$.

Assume that the right-hand side of (4.43) is 1 . Using the above facts and (4.26) it follows that with probability one we have for infinitely many $k$

$$
\begin{aligned}
S_{M_{k}} & >s_{k}\left(\varphi_{M_{k}}+10 / \varphi_{M_{k}}\right) \\
& \geq\left(\sqrt{M_{k} / 2}-O\left(M_{k}^{-1 / 2}\right)\right)\left(\varphi_{M_{k}}+(\log \log k)^{-1 / 2}\right) \geq \sqrt{M_{k} / 2} \varphi_{M_{k}}
\end{aligned}
$$

and thus the left-hand side of (4.43) is also 1. Assume now that the right-hand side of (4.43) is 0 . Then it remains 0 if we replace $\varphi_{M_{k}}$ by $\varphi_{M_{k}}-10 / \varphi_{M_{k}}$ and thus we have almost surely for large enough $k$

$$
\begin{aligned}
S_{M_{k}} & \leq s_{k}\left(\varphi_{M_{k}}-4(\log \log k)^{-1 / 2}\right) \\
& \leq\left(\sqrt{M_{k} / 2}+O\left(M_{k}^{-1 / 2}\right)\right)\left(\varphi_{M_{k}}-4(\log \log k)^{-1 / 2}\right) \\
& \leq \sqrt{M_{k} / 2}\left(\varphi_{M_{k}}-2(\log \log k)^{-1 / 2}\right) .
\end{aligned}
$$

Now if $M_{k} \leq N<M_{k+1}$ then by (4.5), $\alpha \geq 1 / 2$, and $A \leq 10^{-6}$ we have

$$
\left|S_{N}-S_{M_{k}}\right| \leq m_{k+1} \leq \sqrt{M_{k}}(\log \log k)^{-1 / 2} \quad\left(k \geq k_{0}\right),
$$

and thus by (4.44) we have

$$
\begin{aligned}
S_{N} & \leq \sqrt{M_{k} / 2}\left(\varphi_{M_{k}}-2(\log \log k)^{-1 / 2}\right)+\sqrt{M_{k}}(\log \log k)^{-1 / 2} \\
& \leq \sqrt{M_{k} / 2} \varphi_{M_{k}} \leq \sqrt{N / 2} \varphi_{N} \quad\left(N \geq N_{0}\right) .
\end{aligned}
$$


Thus the left-hand side of (4.43) is 0 and the proof of Lemma 4.6 is completed.

Now Theorem 2 follows immediately from Lemmas 4.2-4.6 and the remarks made after the proof of Lemma 4.5.

Remark. Note that we obtained the validity of the test (1.10)-(1.14) for all $\alpha \geq 1 / 2$, regardless whether $\alpha<3 / 2$. For $\alpha \geq 3 / 2$, however, the series (1.14) is equiconvergent with (1.11) (see the computations below) and thus the test (1.10)-(1.14) of Theorem 2 reduces to the Kolmogorov-Feller test.

In conclusion we prove the remarks we made in the Introduction concerning the test (1.10)-(1.14). First we note that if $\varphi_{N}$ satisfies (1.13) then using (1.16) we get for $\alpha>1 / 2,0<A \leq 10^{-6}$, and any $k \geq 1$

$$
\begin{aligned}
\left|\sum_{\nu=k+1}^{\infty} \frac{a_{N, \nu}}{(\log \log N)^{\alpha \nu}} \varphi_{N}^{\nu+2}\right| & \leq \frac{4}{7} \log \log N \sum_{\nu=k+1}^{\infty}\left(96 \sqrt{A}(\log \log N)^{1 / 2-\alpha}\right)^{\nu} \\
& \leq(\log \log N)^{1-(k+1)(\alpha-1 / 2)} \quad\left(N \geq N_{0}\right) .
\end{aligned}
$$

Thus if $\alpha \in\left[c_{k+1}, c_{k}\right)$ then the total contribution of all terms $\nu \geq k+1$ of the sum in the exponential in (1.14) is $O(1)$ and thus for such $\alpha,(1.14)$ is equivalent to (1.18). (If $\alpha \geq 3 / 2$ then the last expression in (4.45) is $O(1)$ for $k=0$ and thus, in this case, the sum in the exponential in (1.14) is $a_{N, 0} \varphi_{N}^{2}+$ $O(1)=\varphi_{N}^{2}+O(1)$ i.e., the test $(1.10)-(1.14)$ reduces to the Kolmogorov-Feller test.) (4.45) also shows that if $\alpha>1 / 2$ and (1.13) holds then the contribution of all terms $\nu \geq 1$ in the sum in $(1.14)$ is $\leq(\log \log N)^{1-(\alpha-1 / 2)}=o\left(\varphi_{N}^{2}\right)$ whence it follows immediately that $\left(\cos n_{k} x\right)$ obeys the ordinary LIL (1.3). Next we prove that for $\alpha=1 / 2$ the test (1.10)-(1.14) implies (1.19). Observe to this end that for $\alpha=1 / 2$ and $\varphi_{N}=c(2 \log \log N)^{1 / 2}, 0 \leq c \leq 2$ the exponent in (1.14) becomes $\exp \left(-f_{N}(c) \log \log N\right)$ where $f_{N}(c)=\sum_{\nu=0}^{\infty} 2^{\nu / 2} a_{N, \nu} c^{\nu+2}$. By (1.16) the total contribution of all terms $\nu \geq 2$ in the last sum is $O(A)$ and thus by (1.15) we get

$$
f_{N}(c)=c^{2}+\frac{1}{3} \sqrt{2 A} c^{3}+O(A),
$$

where the constants implied by the $O$ 's are absolute. Let

$$
c_{1}=1-\frac{1}{6} \sqrt{2 A}+A^{2 / 3}, \quad c_{2}=1-\frac{1}{6} \sqrt{2 A}-A^{2 / 3} .
$$

Substituting into (4.46) we get by a simple calculation

$$
f_{N}\left(c_{1}\right)=1+2 A^{2 / 3}+O(A), \quad f_{N}\left(c_{2}\right)=1-2 A^{2 / 3}+O(A),
$$

and thus $f_{N}\left(c_{1}\right)>1, f_{N}\left(c_{2}\right)<1$ if $A$ is small enough. Hence (1.14) converges for $\varphi_{N}=c_{1}(2 \log \log N)^{1 / 2}$, diverges for $\varphi_{N}=c_{2}(2 \log \log N)^{1 / 2}$, and thus the $\overline{\lim }$ in (1.19) lies between $c_{1}$ and $c_{2}$, completing the proof.

Next we prove the Corollary concerning the upper-lower class behavior of the function $\varphi_{N}$ in (1.22). For simplicity, we shall give the proof for the case $k(\alpha)=1$ (i.e., for $5 / 6 \leq \alpha<1)$ when the sum in (1.22) contains two terms. (This is exactly the third special case listed after the Corollary.) The proof in the general case is the same.

Assume $5 / 6 \leq \alpha<1$; then, as we already observed, (1.14) is equivalent to (1.17). Set

$$
\varphi_{N}=\left(2 \log \log N+c(\log \log N)^{3 / 2-\alpha}+c^{*}(\log \log N)^{2-2 \alpha}+\rho_{\varepsilon}(N)\right)^{1 / 2},
$$


where $c$ and $c^{*}$ are arbitrary constants and $\rho_{\varepsilon}(N)$ is defined by $(1.21)$. Writing $\varphi_{N}$ as

$$
\varphi_{N}=(2 \log \log N)^{1 / 2}\left(1+\frac{c}{2}(\log \log N)^{1 / 2-\alpha}+O\left((\log \log N)^{1-2 \alpha}\right)\right)^{1 / 2}
$$

and using the power series of $(1+x)^{3 / 2}$ we get

$$
\begin{aligned}
& (\log \log N)^{-\alpha} \varphi_{N}^{3} \\
& \quad=\sqrt{8}(\log \log N)^{3 / 2-\alpha}\left(1+\frac{3 c}{4}(\log \log N)^{1 / 2-\alpha}+O\left((\log \log N)^{1-2 \alpha}\right)\right) \\
& =\sqrt{8}(\log \log N)^{3 / 2-\alpha}+\frac{3 c \sqrt{8}}{4}(\log \log N)^{2-2 \alpha}+O(1) .
\end{aligned}
$$

Similarly

$$
\begin{aligned}
(\log \log N)^{-2 \alpha} \varphi_{N}^{4} & =4(\log \log N)^{2-2 \alpha}\left(1+O\left((\log \log N)^{1 / 2-\alpha}\right)\right) \\
& =4(\log \log N)^{2-2 \alpha}+O(1) .
\end{aligned}
$$

Substituting (4.47), (4.48), and (4.49) for the terms of the exponent in (1.17), the exponent becomes

$$
\begin{aligned}
& -\log \log N+\left(-\frac{c}{2}-\frac{\sqrt{2 A}}{3}\right)(\log \log N)^{3 / 2-\alpha} \\
& +\left(-\frac{c^{*}}{2}-\frac{c \sqrt{2 A}}{4}+\frac{5 A}{18}\right)(\log \log N)^{2-2 \alpha}-\frac{\rho_{\varepsilon}(N)}{2}+O(1) .
\end{aligned}
$$

Choosing $c=-2 \sqrt{2 A} / 3$ and $c^{*}=11 A / 9$ the coefficients of $(\log \log N)^{3 / 2-\alpha}$ and $(\log \log N)^{2-2 \alpha}$ in (4.50) become 0 and thus the series (1.17) reduces to

$$
\sum_{N \geq 1}(1+o(1)) \frac{(2 \log \log N)^{1 / 2}}{N} \exp \left\{-\log \log N-\frac{\rho_{\varepsilon}(N)}{2}+O(1)\right\},
$$

which is clearly convergent if $\varepsilon>0$ and divergent if $\varepsilon \leq 0$.

Regarding the first of the special cases listed after the Corollary, its validity clearly follows from the Corollary for $c<2 \sqrt{2 A} / 3$ and $c>2 \sqrt{2 A} / 3$. In the case $c=2 \sqrt{2 A} / 3, \varphi_{N}$ belongs to the lower class for arbitrary $1 / 2<\alpha<3 / 2$ since the coefficient $b_{N, 1}=11 A / 9$ in the sum in (1.22) is positive. (Hence to decide for arbitrary $1 / 2<\alpha<3 / 2$ if

$$
\varphi_{N}=\left(2 \log \log N-\frac{2 \sqrt{2 A}}{3}(\log \log N)^{3 / 2-\alpha}+\frac{11 A}{9}(\log \log N)^{2-2 \alpha}\right)^{1 / 2}
$$

belongs to the upper or lower class (for $1 \leq \alpha<3 / 2$ it is upper class and for $5 / 6 \leq \alpha<1$ it is lower class by special cases 2 and 3 listed after the Corollary) one has to compute coefficient $b_{N, 2}$ in (1.22).)

In conclusion we prove the remark made at the end of the Introduction i.e., we show that if $\left(n_{k}\right)$ is the sequence in Theorem 2 or more generally $\left(n_{k}\right)$ is any sequence satisfying (1.12) for some $A>0, \alpha>0$ then there exists a sequence $\left(m_{k}\right)$ such that $\left|m_{k}-n_{k}\right| \ll k^{3}$ and $\left(\cos n_{k} x\right)$ satisfies the Kolmogorov-Feller test $(1.10)-(1.11)$. In fact, let $I_{k}=\left[n_{k}-4 k^{3}, n_{k}+4 k^{3}\right] \quad(k \geq 1)$; clearly the 
intervals $I_{k}$ are disjoint for $k \geq k_{0}$. Now the desired $\left(m_{k}\right)$ can be constructed by induction as follows. Let $m_{k}=n_{k}$ for $1 \leq k \leq k_{0} a^{2} \mathrm{~d}$ if for some $k \geq k_{0}$, $m_{1}, \ldots, m_{k}$ are already constructed, choose $m_{k+1} \in I_{k+1}$ so that it is different from all numbers of the form $\pm n_{i_{1}} \pm n_{i_{2}} \pm n_{i_{3}}, 1 \leq i_{1}, i_{2}, i_{3} \leq k$. Since the number of such sums is $\leq 8 k^{3}$ and $I_{k+1}$ contains more than $8 k^{3}$ integers, this choice is possible. Clearly, the so constructed sequence $\left(m_{k}\right)$ has the property that for large enough $\nu$ the equation

$$
\nu=m_{k} \pm m_{l} \quad(k>l \geq 1)
$$

has at most one solution. Also, $\left|m_{k}-n_{k}\right| \leq 4 k^{3} \quad(k \geq 1)$ and since (1.12) implies $n_{k} \succ e^{\sqrt{k}}$, we get

$$
\begin{aligned}
\frac{m_{k+1}}{m_{k}} & \geq \frac{n_{k+1}-4(k+1)^{3}}{n_{k}+4 k^{3}}=\frac{n_{k+1}}{n_{k}}\left(1+O\left(k^{3} e^{-\sqrt{k}}\right)\right) \\
& \geq \exp \left(\frac{1}{2 \sqrt{k+1}}\right)\left(1+O\left(k^{-1}\right)\right) \geq\left(1+\frac{1}{4 \sqrt{k}}\right)\left(1-\frac{1}{k}\right) \\
& \geq 1+\frac{1}{5 \sqrt{k}} \quad\left(k \geq k_{0}\right) .
\end{aligned}
$$

Hence by Theorem 3 of [2] $\left(\cos n_{k} x\right)$ satisfies the a.s. invariance principle (1.4) and consequently the Kolmogorov-Feller test (1.10)-(1.11).

\section{REFERENCES}

1. N. K. Bary, A treatise on trigonometric series, Pergamon, New York, London, and Paris, 1964.

2. I. Berkes, On the central limit theorem for lacunary trigonometric series, Anal. Math. 4 (1978), 159-180.

3. _ Nongaussian limit distributions of lacunary trigonometric series, Canad. J. Math. $\mathbf{4 3}$ (1991), 948-959.

4. _ A note on lacunary trigonometric series, Acta Math. Hungar. 57 (1991), 181-186.

5. U. Einmahl and D. M. Mason, Some results on the almost sure behavior of martingales, Proc. Colloq. Soc. J. Bolyai on Limit Theorems in Probab. and Stat., Pécs 1989, North-Holland, Amsterdam (to appear).

6. P. Erdös and I. S. Gál, On the law of the iterated logarithm, Proc. Kon. Nederl. Akad. Wetensch. Ser. A 58 (1955), 65-84.

7. P. Erdős, On trigonometric sums with gaps, Magyar Tud. Akad. Mat. Kut. Int. Közl. 7 (1962), 37-42.

8. W. Feller, The general form of the so-called law of the iterated logarithm, Trans. Amer. Math. Soc. 54 (1943), 373-401.

9. Math. 47 (1946), 631-638.

10. V. F. Gaposhkin, Lacunary series and independent functions, Uspekhi Mat. Nauk 21-6 (1966), 3-82; English transl., Russian Math. Surveys 21-6 (1966), 3-82.

11. T. Murai, On lacunary series, Nagoya Math. J. 85 (1982), 87-154.

12. W. Philipp and W. F. Stout, Almost sure invariance principles for sums of weakly dependent random variables, Mem. Amer. Math. Soc., no. 161, Amer. Math. Soc., Providence, R.I., 1975.

13. _ Invariance principles for martingales and sums of independent random variables, Math. Z. 192 (1986), 253-264.

14. R. Salem and A. Zygmund, On lacunary trigonometric series, Proc. Nat. Acad. Sci. U.S.A. 33 (1947), 333-338. 
15. S. Takahashi, On lacunary trigonometric series, Proc. Japan Acad. 41 (1965), 503-506.

16. __ On the lacunary Fourier series, Tôhoku Math. J. 19 (1967), 79-85.

17. _ On lacunary trigonometric series. II, Proc. Japan Acad. 44 (1968), 766-770.

18. _ On the law of the iterated logarithm for lacunary trigonometric series, Tôhoku Math. J. 24 (1972), 319-329.

19. _ On the law of the iterated logarithm for lacunary trigonometric series. II, Tôhoku Math. J. 27 (1975), 391-403.

20. _ Almost sure invariance principles for lacunary trigonometric series, Tôhoku Math. J. 31 (1979), 439-451.

21. A. Zygmund, Trigonometric series. I-II, Cambridge Univ. Press, Cambridge, 1959.

Mathematical Institute of the Hungarian Academy of Sciences, H-1053 Budapest, ReÁltanoda U. 13-15, Hungary

E-mail address: h1127ber@ella.hu 